\title{
SOME APPLICATIONS OF NEVANLINNA THEORY TO MATHEMATICAL LOGIC: IDENTITIES OF EXPONENTIAL FUNCTIONS
}

\author{
BY
}

C. WARD HENSON AND LEE A. RUBEL

Dedicated to the memory of Rolf Nevanlinna

\begin{abstract}
In this paper we study identities between certain functions of many variables that are constructed by using the elementary functions of addition $x+y$, multiplication $x \cdot y$, and two-place exponentiation $x$. For a restricted class of such functions, we show that every true identity follows from the natural set of eleven axioms. The rates of growth of such functions, in the case of a single independent variable $x$, as $x \rightarrow \infty$, are also studied, and we give an algorithm for the Hardy relation of eventual domination, again for a restricted class of functions. Value distribution of analytic functions of one and of several complex variables, especially the Nevanlinna characteristic, plays a major role in our proofs.
\end{abstract}

Introduction. To make precise the questions we consider, it is necessary to introduce a formal language containing the expressions which define the elementary functions to be studied. This language has variables $x_{1}, x_{2}, \ldots$, a set $S$ of constants (representing a given set of fixed numbers) and function symbols for addition, multiplication and exponentiation. The terms (that is, the function-defining expressions) are obtained inductively starting with the variables and constants and continuing according to the rule: if $t$ and $s$ are terms, then so are $(t+s),(t \cdot s)$ and $\left(t^{s}\right)$. (We follow some standard conventions for dropping parentheses and $\cdot$ below.) Let us refer to these as the exponential terms over $S$.

In most cases considered here the constants in $S$ represent positive real numbers. In such a case, every term $t$ represents a function which is defined for all positive real values of the variables which occur in it. We write $t \equiv s$ to mean that $t$ and $s$ are terms which define exactly the same function (for positive real values of the variables appearing in $t$ or $s$ ). It is necessary to distinguish between this function equality relation $t \equiv s$ and a formal expression of equality between $t$ and $s$, which we write in the form $t=s$. The relation $t \equiv s$ is a mathematical relation between certain elementary functions; the expression $t=s$ is a purely formal expression which is susceptible to formal proof within various axiomatic systems.

A closely related topic concerns the orders of growth of functions of one variable defined by exponential terms. If $t$ and $s$ are such terms which contain only the single

Received by the editors February 16, 1983.

1980 Mathematics Subject Classification. Primary 03B25, 03C05, 30D35, 32A22. 
variable $x$, we write $t \leqslant s$ to mean that there exists a number $a>0$ in $\mathbf{R}$ such that if $x \geqslant a$, then the function value defined by $t$ at $x$ is $\leqslant$ the corresponding function value defined by $s$. (That is, $t \leqslant s$ means that $s$ defines a function which eventually dominates the function defined by $t$.)

Investigation of these relations $\equiv$ and $\preccurlyeq$ has a long history. For example, G. $\mathrm{H}$. Hardy [HAR] showed that the eventual dominance relation $\preccurlyeq$ is a linear ordering on a large class of elementary functions including all the ones defined by exponential terms over R. (Hardy's class is closed under log as well as under addition, multiplication and exponentiation. Such a linear ordering result for a much larger class of functions has recently been obtained by M. Boshernitzan [BOS].) Remarkably, $\preccurlyeq$ is a well-ordering on the functions defined by one-variable, exponential terms over the set $\mathbf{N}$ of positive integers, as shown by A. Ehrenfeucht [EHR]. It is an open problem to determine the ordinal of this well-ordering. Also it is not known if there exists an algorithm for deciding whether $t \preccurlyeq s$ holds, where $t$ and $s$ are one-variable exponential terms over $\mathbf{N}$. In this paper we give an algorithm for the relation $t \preccurlyeq s$, where $t$ and $s$ come from a restricted class of exponential terms. Earlier, H. Levitz gave a noneffective analysis of $\preccurlyeq$ on this class of terms and showed that in this case the order type is $\varepsilon_{0}$ [LEV].

For exponential terms $t, s$ (in many variables) over N, A. Macintyre [MAC] has given an algorithm which decides whether or not $t \equiv s$ holds. Earlier D. Richardson [RIC II] had given such an algorithm for one-variable terms. A central problem for exponential terms over $\mathbf{N}$ has been A. Tarski's High School Algebra Conjecture. (See [MAR or TAW].) This asserts that if $t, s$ are exponential terms over $\mathbf{N}$ and $t \equiv s$, then the formal identity $t=s$ can be derived from certain elementary identities plus numerical axioms which give the facts of addition, multiplication and exponentiation for positive integers (e.g. $2^{3}=8$ ). The identities proposed as basic axioms by Tarski express the fundamental laws of addition, multiplication and exponentiation which are taught in basic mathematics education:

$$
\begin{aligned}
x+(y+z) & =(x+y)+z, & x^{y+z} & =x^{y} x^{z}, \\
x+y & =y+x, & x^{y z} & =\left(x^{y}\right)^{z}, \\
x(y z) & =(x y) z, & (x y)^{z} & =x^{z} \cdot y^{z}, \\
x y & =y x, & x^{1} & =x, \\
x(y+z) & =x y+x z, & 1^{x} & =1 .
\end{aligned}
$$

Tarski's conjecture for the full class of exponential terms has been shown to be false by A. Wilkie [WIL II]. In this paper Wilkie also proves a positive result toward a version of Tarski's conjecture. He expands the formal language of the original problem by adding a function symbol $f$ for each polynomial $p\left(x_{1}, \ldots, x_{n}\right)$ which is positive for all positive values of $x_{1}, \ldots, x_{n}$. To the Tarski identities in the larger language he adds all true polynomial equations (using the new function symbols). Wilkie's main result asserts that if $t$ and $s$ are exponential terms in the original language and if $t \equiv s$, then there is a proof of the equation $t=s$ from the enlarged system of axioms in the larger language. In this paper we show that when the class of exponential terms is suitably restricted, Tarski's conjecture is true without passage to the larger language. 
In a final section (\$5) we treat a slightly different class of exponential functions - those with exponentials restricted to constant bases and allowing complex numbers to appear as constants. Let $\exp (\cdot)$ be a function symbol corresponding to exponentiation with the constant base $e$. Then $\Sigma$ is the class of formal terms defined starting with variables and constants and then containing $(t+s),(t \cdot s)$ and $\exp (t)$ for any terms $t$ and $s$. Each term $t$ in $\Sigma$ defines a holomorphic function on $\mathbf{C}^{n}$, where $n$ is the number of variables in $t$. We prove a form of Tarski's Conjecture for $\Sigma$ : if $t, s \in \Sigma$ and $t \equiv s$, then the formal equality $t=s$ is derivable from a list of elementary identities together with numerical axioms giving the facts of addition, multiplication and exponentiation in $\mathbf{C}$ (Theorem 5.2). This result was proved independently by van den Dries [VDD] and, for one-variable terms, by Wilkie [WIL I]. From this follows a similar completeness theorem for identities involving,$+ \cdot$, sin and cos. Finally we settle positively a conjecture of S. Schanuel concerning the class $\Sigma$, which asserts essentially that it is "closed under logarithms". Specifically, we show that if $t \in \Sigma$ and the function on $\mathbf{C}^{n}$ defined by $t$ is nowhere equal to 0 , then there exists a term $s$ in $\Sigma$ so that $t \equiv \exp (s)$. In particular, for example, this shows that there is no term in $\Sigma$ which defines the function on $\mathbf{C}$ equal to $\exp (\sin (z) / z)$.

The methods used by Wilkie to study Tarski's conjecture are those of differential algebra, while van den Dries uses a direct algebraic approach. Therefore their results apply somewhat more broadly than ours to a class of "exponential rings". Moreover, it seems likely that most of the results we give here concerning the provability of identities could be proved using an algebraic or differential algebraic approach. Several people have suggested this possibility to us; to our knowledge no detailed argument has yet been provided. An exception to this possibility seems to be our positive solution of Schanuel's conjecture, for which no direct algebraic approach has been suggested.

To our knowledge, this is the first time value distribution theory (Nevanlinna theory) has been applied to questions in mathematical logic. A brief example will show why this theory might be useful in studying identities of exponential functions. Suppose, for example, $f, g$ and $h$ are entire functions satisfying the identity $e^{f}+e^{g}$ $=e^{h}$. Letting $F=f-h$ and $G=g-h$ we see $e^{F}+e^{G}=1$. Therefore $e^{F}$ omits both of the values 0 and 1. By the Little Picard Theorem it follows that $F$, and hence also $G$, are constants.

The Nevanlinna characteristic provides the tool for applying similar arguments in a much wider setting.

The results proved here concern a restricted class $\varrho(S)$ of exponential terms. This class contains the variables $x_{1}, x_{2}, \ldots$ and constants from $S$; also it contains $(t+s)$ and $(t \cdot s)$ whenever $t, s$ are terms in $\mathcal{L}(S)$; the closure under exponentiation is restricted, however; we require that $\mathscr{L}(S)$ contain $\left(t^{s}\right)$ whenever $s$ is in $\mathscr{L}(S)$ and $t$ is a constant $>1$ from $S$ or a variable $x_{j}$ or a power of a variable $x_{j}^{a}$ for some constant $a$ from $S$. In general we require that the set $S$ of constants should represent numbers that are $\geqslant 1$. Thus, for example, $\mathcal{L}(\mathbf{N})$ does contain

$$
\left(x_{1}^{2}\right)^{x_{2}+x_{3}} \cdot 3^{x_{2}^{x_{1}}}
$$


but it does not contain the exponential term $\left(x_{1}+x_{2}\right)^{x_{3}}$ because the base of the exponential in it contains + .

Our main results for $\mathcal{L}(S)$ are given in $\S \S 3$ and 4 . We show that Tarski's High School Algebra Conjecture is true for terms in $\mathcal{L}(S)$ (Corollary 3.5). This is derived from a Normal Form Theorem for $\mathcal{L}(S)$ (Theorem 3.4). Also we give an algorithm which decides the eventual dominance relation $t \preccurlyeq s$ for one-variable terms in $\mathcal{L}(\mathbf{N})$ (Theorem 4.1).

The one-variable terms in $\mathcal{L}(\mathbf{N})$ were studied by $\mathrm{H}$. Levitz [LEV], who showed that the ordinal of the ordering $\preccurlyeq$ on them is exactly $\varepsilon_{0}$.

The technical details of our approach to these results are given in $\S \S 1$ and 2 . In $\S 1$ we give a close analysis of the orders of growth of functions defined by one-variable terms in $\mathcal{L}(S)$. (See especially Theorem 1.7.) We also treat the orders of growth of many-variable terms in $\mathscr{L}(S)$ when one of the variables tends to $\infty$ and the others are held fixed. The main technical fact used later is a lemma on exponential growth (Corollary 1.15).

Our main innovation in treating these problems lies in the use of the Nevanlinna characteristic. This is described in $\S 2$. Each term in $\mathscr{L}(S)$ gives a holomorphic function when its variables are taken to range over the complex domain $\Omega$, which is the complex plane slit along the negative real axis $\Omega=\mathbf{C} \backslash(-\infty, 0]$. The domain $\Omega^{n}$ is biholomorphically equivalent to the polydisc $\mathbf{D}^{n}, \mathbf{D}=\{z \in \mathbf{C}|| z \mid<1\}$. We use this shifting of viewpoint together with the Nevanlinna characteristic for meromorphic functions on the polydisc to handle the connection between many-variable and one-variable terms in $\mathcal{L}(S)$.

At the beginning of $\$ 2$ we give an essentially axiomatic description of the Nevanlinna characteristic (see properties (C2.0)-(C2.6)). In addition we must make an explicit estimate for the Nevanlinna characteristic of certain functions defined by terms in $\mathcal{L}(S)$ (Lemmas 2.2 and 2.3). The object of our use of Nevanlinna theory lies in a linear independence lemma (Lemma 2.1) which is a version of a result proved for functions of one variable by Hiromi and Ozawa [HIO]. This result, together with Lemma 2.2, gives all the consequences of Nevanlinna theory which are used in the main results of the paper in $\S 3$.

In $§ 3$ we not only prove Tarski’s High School Algebra Conjecture for $\mathcal{L}(S)$, which is closed only under a restricted form of exponentiation, but also for two other classes of terms. One of these, $\mathcal{E}(S)$, is the class of all exponential terms over $S$ in which only exponentiation occurs (and no addition or multiplication). When $S=\varnothing$ this was proved earlier in the thesis of C. Martin [MAR] by a proof-theoretic argument. Here it turns out that all true identities are provable from the identity $\left(x^{y}\right)^{z}=\left(x^{z}\right)^{y}$. We also treat the class $\mathcal{G} \mathscr{N}(S)$ of all exponential terms in which only exponentiation and multiplication occur. Both $\mathscr{E}(S)$ and $\mathscr{E} \Re(S)$ are in fact closely related to $\mathcal{L}(S)$.

The methods of this paper should be useful in treating other classes of elementary functions and their identities. For example, consider the class of terms $\mathcal{R}(S) \supseteq \mathcal{L}(S)$, where $\mathcal{R}(S)$ is defined to be closed under formation of terms $(t+s)$ and $(t \cdot s)$ and also $\left(p^{t}\right)$ where $t, s$ are any terms in $R(S)$ and $p$ is any polynomial with coefficients 
in $S$. The counterexample found by Wilkie to the Tarski Conjecture for the full class of exponential terms over $\mathbf{N}$ is "just outside" $R(\mathbf{N})$. It seems possible that the methods of this paper can be used to prove Tarski's Conjecture for the classes $\Re(S)$.

More generally one hopes to discover, where possible, simple systems of identities for many classes of elementary functions from which it is possible to formally derive all true identities. (Some limiting undecidability results have been proved by $D$. Richardson [RIC I].)

We gratefully acknowledge helpful conversations, correspondence and preprints from many people, including M. Boshernitzan, A. Macintyre, W. Stoll, L. van den Dries and A. Wilkie.

1. Orders of growth of certain exponential terms. Here we define precisely the restricted class of exponential terms which will be studied in this paper and we give an explicit analysis of the eventual dominance ordering $\preccurlyeq$ on the l-variable terms in this class. The terms we consider are very closely related to the ones studied by $\mathrm{H}$. Levitz [LEV]. Our main generalization is to allow an arbitrary set $S$ of positive, real constants. The case treated by Levitz corresponds to letting $S=\mathbf{N}=\{1,2,3, \ldots\}$. We are also interested in the special cases where $S=\mathbf{R}^{+}=\{r \in \mathbf{R} \mid r>0\}$ and $S=\mathbf{R}^{\geqslant 1}=\{r \in \mathbf{R} \mid r \geqslant 1\}$.

Fix a set $S$ of positive real numbers which contains 1 and satisfies the closure condition:

$$
a, b \in S \Rightarrow a+b, a \cdot b, a^{b} \in S .
$$

Definition 1.1. The Levitz class of exponential terms (with constants from $S$ ), which we denote by $\mathcal{L}(S)$, is the smallest class $C$ of formal terms which satisfies:

(1) $巳$ contains a constant for each element of $S$ and contains the variables $x_{1}, x_{2}, x_{3}, \ldots$

(2) If $t, s \in \mathcal{C}$, then $t+s \in \mathcal{C}$.

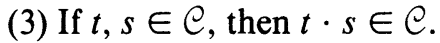

(4) If $t \in \mathcal{C}$ and $B \in S$ satisfies $B>1$, then $B^{t} \in \mathcal{C}$.

(5) If $t \in \mathcal{C}$ then for each variable $x_{n}, x_{n}^{t} \in \mathcal{C}$; moreover, for each $a \in S$ the term $\left(x_{n}^{a}\right)^{t}$ is also in $\mathrm{C}$.

Note. (1) While $\left(x_{n}^{a}\right)^{t}$ is formally different from $x_{n}^{a \cdot t}$, they do represent the same function over R. Our reason for including both terms in $\varrho(S)$ is to allow the Normal Form Theorem proved below to be expressed in a simpler way.

(2) If we restrict to a single variable $x_{1}$, the terms in $\mathcal{L}(\mathbf{N})$ are essentially the same as the ones studied by Levitz [LEV]; this is our reason for calling $\mathcal{L}(S)$ the Levitz class in general.

(3) The constants which can appear in terms in $\mathcal{L}(S)$ are all positive; no subtraction or division operations are permitted in $\mathcal{L}(S)$ either.

DEFINITION 1.2. $\mathcal{L}_{1}(S)$ is the class of terms in $\mathcal{L}(S)$ which only involve the single variable $x_{1}$.

(For convenience we will write $x$ instead of $x_{1}$ when considering terms in $\mathcal{L}_{1}(S)$.)

In this section we give an explicit analysis of the orders of growth of the terms in $\mathfrak{E}_{1}(S)$ and prove a Normal Form Theorem. This is similar to the analysis done by 
Levitz [LEV], but we give a constructive (and very simple) procedure for obtaining the normal form of a given term. In $\S 4$ we will show that this provides a decision procedure for the eventual dominance relation $\preccurlyeq$ on $\mathcal{L}_{1}(\mathbf{N})$ and some other classes of this kind.

Each term in $\mathcal{L}_{1}(S)$ has an interpretation as a function on $\mathbf{R}^{+}=\{r \in \mathbf{R} \mid r>0\}$. We define $t \equiv s$ (for $t, s \in \mathcal{L}_{1}(S)$ ) to mean that $t$ and $s$ represent the same function on $\mathbf{R}^{+}$. We write $t \preccurlyeq s$ to mean that there is an $a>0$ so that on the interval $a \leqslant x<\infty$ the function represented by $t$ is $\leqslant$ the function represented by $s$. We define $t \prec s$ to mean $(t \preccurlyeq s$ and $t Z s)$.

Since the terms in $\mathcal{L}_{1}(S)$ define real-analytic functions on $\mathbf{R}^{+}$, we see that $(t \preccurlyeq s$ and $s \preccurlyeq t$ ) implies $t \equiv s$. G. H. Hardy [HAR] showed that $\preccurlyeq$ actually defines a linear ordering: for any $t, s \in \mathcal{L}_{1}(S)$ exactly one of the conditions $t \prec s$ or $t \equiv s$ or $s \prec t$ must hold. Hardy showed this for a much larger class of terms, including the class of exponential terms. (See the Introduction.) Ehrenfeucht [EHR] observed that on this class the relation $\preccurlyeq$ is actually a well-ordering. In particular, this is true on $\varrho_{1}(\mathbf{N})$. This is a remarkable fact, but it will play no role in this paper.

The class $\mathscr{H}$ of terms treated by Hardy is closed under division, subtraction and logarithms, as well as multiplication, addition and exponentiation. He showed that if $f \in \mathscr{H}$ then $f(x)$ is eventually monotone as $x$ approaches infinity, so that $f(x)$ approaches a (possibly infinite) limit there.

It follows from this that if $t, s \in \mathcal{L}_{1}(S)$ then such expressions as $t / s, \log (t) / \log (s)$, etc. all approach limits as $x \rightarrow \infty$.

DeFInItion 1.3. We define for $t \in \varrho_{1}(S)$, the concept $t$ is an atom by induction on the number of symbols in the formal term $t: t$ is an atom if it is of the form $u_{1}^{t_{1}} u_{2}^{t_{2}} \cdots u_{n}^{t_{n}}(n \geqslant 1)$ where:

(i) each $u_{j}$ is either a constant $B>1$ in $S$ or of the form $x^{a}$ for some $a \in S$;

(ii) each $t_{j}$ is either 1 or an atom;

(iii) if $u_{j}$ is a constant, then $t_{j}$ must be an atom;

(iv) if $u_{i}, u_{j}$ are constants and $i \neq j$, then $t_{i}, t_{j}$ are distinct terms;

(v) if $u_{i}, u_{j}$ are both of the form $x^{a}$ (but not necessarily with the same $a$ ) and $i \neq j$, then $t_{i}, t_{j}$ are distinct terms;

(vi) $u_{n}^{t_{n}} \preccurlyeq \cdots \leqslant u_{2}^{t_{2}} \leqslant u_{1}^{t_{1}}$.

DEFINITION 1.4. An atom $t=u_{1}^{t_{1}} \cdots u_{n}^{t_{n}}$ is exponential if no $t_{j}$ is the constant symbol 1 .

Definition 1.5. Let $t=u_{1}^{t_{1}} \cdots u_{n}^{t_{n}}$ and $s=v_{1}^{s_{1}} \cdots v_{m}^{s_{m}}$ be atoms. Define Lex $(t, s)$ $\Leftrightarrow$ the sequence $\left(t_{1}, u_{1}, t_{2}, u_{2}, \ldots, t_{n}, u_{n}\right)$ is lexicographically earlier than the sequence $\left(s_{1}, v_{1}, s_{2}, v_{2}, \ldots, s_{m}, v_{m}\right)$, where the ordering $\prec$ is used at each coordinate. (That is, either the first sequence is a proper initial segment of the second, or, at the first coordinate where the corresponding entries do not define the same function on $\mathbf{R}^{+}$, the entry of the first sequence is $\prec$ the corresponding entry of the second sequence. For example, $\operatorname{Lex}(t, s)$ holds if $t_{1} \equiv s_{1}$ and $u_{1} \prec v_{1}$ or if $t_{1} \equiv s_{1}, u_{1} \equiv v_{1}$ and $t_{2} \prec s_{2}$.)

Note that Lex $(t, s)$ defines a linear ordering on atoms in the sense that for any atoms $t$ and $s$ as above, either $\operatorname{Lex}(t, s)$ or $\operatorname{Lex}(s, t)$ or $n=m$ and for each $1 \leqslant j \leqslant n, t_{j} \equiv s_{j}$ and $u_{j} \equiv v_{j}$. 
The particular restrictions which appear in our definition of "atom" may be easier to accept after considering identities such as these:

$$
(A B)^{t} \equiv A^{t} \cdot B^{t}, \quad\left(x^{a+b}\right)^{t} \equiv\left(x^{a}\right)^{t} \cdot\left(x^{b}\right)^{t}, \quad\left(x^{1}\right)^{t} \equiv x^{t} .
$$

The restrictions given in the definition turn out to be adequate to give a Normal Form Theorem.

According to Definition 1.3 every atom is a nontrivial term containing $x_{1}$. In particular no constant is an atom. To see the relation between general terms and atoms, see Proposition 1.9(2).

LEMMA 1.6. If $t$ is an atom in $\mathcal{L}_{1}(S)$, then for some $a \in S, x^{a} \preccurlyeq t$.

Proof. By induction on the number of symbols in $t$. If $t$ has a factor of the form $\left(x^{a}\right)^{1}$ then we are clearly done. If not, then $t$ has a factor of the form $u^{s}$ where $s$ is an atom and $u$ is $x^{b}(b \in S)$ or $B(B \in S$ and $B>1)$. In that case there exist $B>1$ so $1<B \preccurlyeq u$ and $a \in S$ so $x^{a} \leqslant s$ by the induction hypothesis. Hence $x^{a} \leqslant B^{x^{a}} \preccurlyeq u^{s}$ $\preccurlyeq t$.

Consider a factor $u^{s}$ which may occur in an atom $t$. If $s$ is 1 then $u^{s}$ is $\left(x^{a}\right)^{1}$ for some $a \in S$, and represents a function of polynomial growth. Otherwise $x^{a} \leqslant s$ for some $a \in S$ (by Lemma 1.6) and $u$ is either a constant $B>1$ or $x^{b}$ for some $b \in S$, so that $u^{s}$ represents a function with exponential growth ( $A^{x^{u}} \preccurlyeq u^{s}$ for some $A>1$ and some $a>0$ ).

Note also that an atom $t=u_{1}^{t_{1}} \cdots u_{n}^{t_{n}}$ can contain at most one factor $u_{j}^{t_{j}}$ of polynomial growth. Moreover, if such a factor occurs, then it must be the factor $u_{n}^{t_{n}}$ of slowest growth.

THEOREM 1.7. Let $t$ and $s$ be atoms in $\mathcal{L}_{1}(S)$.

(1) If $\operatorname{Lex}(t, s)$ holds, then $x^{c} \cdot t \preccurlyeq s$ for some $c>0$. (c need not be in $S$, but it can be taken to be $b-a$ where $a<b$ and $a, b \in S$.)

(2) If $t \equiv s$, then $t$ and $s$ are identical as terms.

(3) If $t$ and $s$ are exponential and $\operatorname{Lex}(t, s)$ holds, then $B^{x^{u}} \cdot t \preccurlyeq s$ for some $B>1$ and some $a \in S$. ( $B$ need not be in $S$, but it can be taken to be a rational number.)

Proof. We prove (1), (2) and (3) simultaneously by a lengthy induction on the total number of symbols in the pair of terms $t, s$. We write $t$ as $u_{1}^{t_{1}} \ldots u_{n}^{t_{n}}$ and $s$ as $v_{1}^{s_{1}} \ldots v_{m}^{s_{m}}$ as in the definition of "atom". Also write $u_{j}$ as $x^{a_{j}}$ or $A_{j}$ and $v_{j}$ as $x^{b_{j}}$ or $B_{j}$, as appropriate. ( $a_{j}, b_{j} \in S ; A_{j}, B_{j} \in S$ and $>1$.)

The basis step of this induction is where $t, s$ are each of the form $B^{x}$ for some $B>1$ in $S$ or the form $\left(x^{a}\right)^{1}$ or $\left(x^{a}\right)^{x}$ for some $a \in S$. In these cases (1), (2) and (3) are trivial.

Now fix atoms $t, s$ and assume the induction hypothesis that (1), (2) and (3) hold for all pairs of atoms $t^{\prime}, s^{\prime}$ which have fewer symbols than $t, s$.

PART (a): We prove first (3) for $t, s$. Assume $\operatorname{Lex}(t, s)$ holds and $t, s$ are exponential atoms.

Case 1. Suppose $\left(t_{1}, u_{1}, \ldots, t_{n}, u_{n}\right)$ is a proper initial segment of $\left(s_{1}, v_{1}, \ldots, s_{m}, v_{m}\right)$. That is, $n<m$ and for each $j \leqslant n$ we have $t_{j} \equiv s_{j}$ and $u_{j} \equiv v_{j}$. Then $s$ has at least one extra factor and it is a factor of exponential growth. Hence the conclusion of (3) is true. 
Case 2. Suppose $t_{1} \prec s_{1}$. We may apply (1) to $t_{1}, s_{1}$ and to $s_{1}, t_{1}$. This plus the linearity of our lexicographic ordering shows that $\operatorname{Lex}\left(t_{1}, s_{1}\right)$ must hold. Hence there exists $c>0$ as in (1) so that $x^{c} \cdot t_{1} \preccurlyeq s_{1}$. Choose $B>1$ so that $B \prec u_{1}$ and use the lemma to choose $a \in S$ so that $x^{a} \preccurlyeq t_{1}$. Then for some $\alpha>0, u_{1}^{n+1} \preccurlyeq x^{\alpha} \preccurlyeq v_{1}^{x^{c}}$ since $u_{1}$ and $v_{1}$ are constant or constant powers of $x$. Hence

$$
\begin{aligned}
B^{x^{a}} \cdot t & \equiv B^{x^{a}} \cdot u_{1}^{t_{1}} \cdots u_{n}^{t_{n}} \preccurlyeq\left(u_{1}^{t_{1}}\right)^{n+1} \\
& \equiv\left(u_{1}^{n+1}\right)^{t_{1}} \preccurlyeq v_{1}^{x^{c} \cdot t_{1}} \preccurlyeq v_{1}^{s_{1}} \preccurlyeq s
\end{aligned}
$$

and we see that (3) is true.

Case 3. Suppose $t_{1} \equiv s_{1}$ and $u_{1} \prec v_{1}$. Since $t$ and $s$ are exponential, the terms $t_{1}$ and $s_{1}$ must be atoms. By (2) of the induction hypothesis, they must be identical.

First consider the case where $n=1$ and $t$ consists of a single factor $u_{1}^{t_{1}}$. Choose a constant $B>1$ so that $B \cdot u_{1} \prec v_{1}$, using the fact that $u_{1}$ and $v_{1}$ are constants or powers of $x$. Use the lemma to choose $a \in S$ with $x^{a} \preccurlyeq t_{1}$. Then

$$
B^{x^{a}} \cdot t \equiv B^{x^{a}} \cdot u_{1}^{t_{1}} \preccurlyeq\left(B u_{1}\right)^{t_{1}} \preccurlyeq v_{1}^{s_{1}} \preccurlyeq s .
$$

Thus we may assume $n>1$. Apply the induction hypothesis (3) to the pair of terms $u_{1}^{t_{1}}$ and $v_{1}^{s_{1}}$, obtaining $A>0$ and $a \in S$ such that $A^{x^{a}} \cdot u_{1}^{t_{1}} \preccurlyeq v_{1}^{s_{1}}$. Take $1<B<A$. We claim that $B^{x^{a}} \cdot t \preccurlyeq v_{1}^{s_{1}}$ (and therefore $\preccurlyeq s$ as needed.) Consider

$$
\begin{aligned}
\frac{\log \left(B^{x^{a}} \cdot t\right)}{\log \left(v_{1}^{s_{1}}\right)} & =\frac{\log \left(B^{x^{a}} \cdot u_{1}^{t_{1}} \cdots u_{n}^{t_{n}}\right)}{\log \left(v_{1}^{s_{1}}\right)} \\
& =\frac{\log \left(B^{x^{a}} \cdot u_{1}^{t_{1}}\right)}{\log \left(v_{1}^{s_{1}}\right)}+\sum_{j=2}^{n} \frac{t_{j} \log \left(u_{j}\right)}{s_{1} \log \left(v_{1}\right)} .
\end{aligned}
$$

The choice of $B$ and $a$ insure that the first term of this sum approaches a limit which is strictly less than 1 . We will show that the rest of the terms approach 0 , which gives the desired result.

Fix $j \geqslant 2$. The linearity of our lexicographic ordering insures that either $\operatorname{Lex}\left(u_{1}^{t_{1}}, u_{j}^{t_{j}}\right)$ holds or $u_{1}^{t_{1}} \equiv u_{j}^{t_{j}}$ or $\operatorname{Lex}\left(u_{j}^{t_{j}}, u_{1}^{t_{1}}\right)$ holds. Since we have assumed $u_{j}^{t_{j}} \preccurlyeq u_{1}^{t_{1}}$, induction hypothesis (3) rules out the first possibility. The second is also ruled out, since the induction hypothesis (2) would imply that $u_{1}^{t_{1}}$ and $u_{j}^{t_{j}}$ were identical, which contradicts the definition of "atom". Therefore $\operatorname{Lex}\left(u_{j}^{t_{j}}, u_{1}^{t_{1}}\right)$ must hold.

Suppose $t_{j} \prec t_{1}$. Applying the induction hypotheses (1) and (3) to both pairs $t_{1}, t_{j}$ and $t_{j}, t_{1}$ yields that $x^{c} \cdot t_{j} \preccurlyeq t_{1}$ for some $c>0$. In particular,

$$
\frac{t_{j} \log \left(u_{j}\right)}{s_{1} \log \left(v_{1}\right)}=\frac{t_{j} \log \left(u_{j}\right)}{t_{1} \log \left(v_{1}\right)} \leqslant \frac{\log \left(u_{j}\right)}{x^{c} \log \left(v_{1}\right)} \leqslant \frac{\log \left(u_{j}\right)}{x^{c} \log \left(u_{1}\right)} .
$$

Since $u_{1}$ and $u_{j}$ are either constants $>1$ or powers of $x$, this last term approaches 0 as $x$ tends to $\infty$.

Otherwise $t_{j} \equiv t_{1}$ and $u_{j} \prec u_{1}$. Induction hypothesis (2) implies that $t_{j}$ and $t_{1}$ are identical terms. Since $t$ is an atom, this yields that $u_{j}$ is a constant $>1$ while $u_{1}$ is $x^{\alpha}$ 
for some $\alpha \in S$. But then

$$
\frac{t_{j} \log \left(u_{j}\right)}{s_{1} \log \left(v_{1}\right)} \equiv \frac{\log \left(u_{j}\right)}{\log \left(v_{1}\right)} \preccurlyeq \frac{\log \left(u_{1}\right)}{\log \left(v_{1}\right)},
$$

and this last term approaches 0 as $x$ tends to $\infty$.

Case 4. Suppose $t_{1} \equiv s_{1}$ and $u_{1} \equiv v_{1}$. The induction hypothesis (2) implies that $t_{1}$ and $s_{1}$ are identical. Also $u_{1}, v_{1}$ must clearly be identical. Since $\operatorname{Lex}(t, s)$ holds, we must have $n>1$ and $m>1$. Let $t^{\prime}=u_{2}^{t_{2}} \cdots u_{n}^{t_{n}}$ and $s^{\prime}=v_{2}^{s_{2}} \cdots v_{m}^{s_{m}}$. Evidently Lex $\left(t^{\prime}, s^{\prime}\right)$ holds, so the induction hypothesis (3) yields $B>1$ and $a \in S$ such that $B^{x^{a}} \cdot t^{\prime} \preccurlyeq s^{\prime}$. But this gives $B^{x^{a}} \cdot t \preccurlyeq s$, as desired.

PART (b). Now we prove (1) for $t, s$, again assuming (1), (2), (3) for all pairs with fewer symbols. Suppose $\operatorname{Lex}(t, s)$ holds. Write $t=t^{\prime} \cdot x^{a}$ and $s=s^{\prime} \cdot x^{b}$ where each of $t^{\prime}, s^{\prime}$ is an exponential atom or 1 . (If $t$, for example, is exponential then we take $a$ to be 0 ; in that case $t$ is identical to $t^{\prime}$.) We may apply (3) to the pair $t^{\prime}, s^{\prime}$. Note that $\operatorname{Lex}\left(s^{\prime}, t^{\prime}\right)$ cannot hold, since $\operatorname{Lex}(t, s)$ does hold. If $\operatorname{Lex}\left(t^{\prime}, s^{\prime}\right)$ is true, then for some $d \in S$ and $B>1$ we have $B^{x^{d}} \cdot t^{\prime} \leqslant s^{\prime}$. Then the exponential growth of $B^{x^{d}}$ insures that for any $c>0, x^{c} \cdot t \preccurlyeq s$. (In fact,

$$
A^{x^{d}} \cdot t \preccurlyeq s
$$

for $1<A<B$, in this case.)

If $\operatorname{Lex}\left(t^{\prime}, s^{\prime}\right)$ and $\operatorname{Lex}\left(s^{\prime}, t^{\prime}\right)$ both fail, then the induction hypothesis (2) insures that $t^{\prime}$ and $s^{\prime}$ have exactly the same factors, and hence $t^{\prime}$ is identical to $s^{\prime}$. Since Lex $(t, s)$ holds, this means $a<b$. Thus if we set $c=b-a$, then $x^{c} \cdot t \preccurlyeq s$ as desired.

PART (c). Now we prove (2) for $t, s$. We assume (1), (2), (3) for all pairs of atoms with fewer symbols; Part (b) gives that (1) is valid for both pairs $t, s$ and $s, t$. This shows that if $t \equiv s$, then $\operatorname{Lex}(t, s)$ and $\operatorname{Lex}(s, t)$ must both fail. Hence $n=m$ and for all $j=1, \ldots, n, t_{j} \equiv s_{j}$ and $u_{j} \equiv v_{j}$. Induction hypothesis (2) yields that $t_{j}$ and $s_{j}$ must be identical for each $j$. Also $u_{j}$ and $v_{j}$ must be identical, since they are constants or of the form $x^{a}$. That is, $t$ and $s$ are identical.

This completes the proof of the theorem.

COROLlary 1.8. Let $t$ and $s$ be atoms in $\varrho_{1}(S)$. Exactly one of the following conditions is true.

(1) For some $B>1$ and $a \in S, B^{x^{a}} \cdot t \preccurlyeq s$.

(2) For some $B>1$ and $a \in S, B^{x^{a}} \cdot s \preccurlyeq t$.

(3) For some $a, b, x^{b} \cdot t \equiv x^{a} \cdot s$. (Here $a, b$ are equal to 0 or are in $S$.)

Proof. Theorem 1.7 shows that if (1) and (2) both fail, then $t$ and $s$ have the same exponential factors. From this one gets (3) immediately.

The next proposition shows how general terms in $L_{1}(S)$ are related to atoms. It is proved using very simple algebraic manipulations.

Proposition 1.9. (1) Let $t_{1}, \ldots, t_{n}$ be terms in $\mathcal{L}_{1}(S)$ and let $u_{1}, \ldots, u_{n}$ be allowed bases (i.e. each $u_{j}$ is either a constant $B>1$ from $S$ or a power $x^{a}$ for some $a \in S$ ). 
Then there exists a constant $c$ in $S$ and an atom $t$ in $\mathcal{L}_{1}(S)$ so that

$$
c \cdot t \equiv u_{1}^{t_{1}} \cdots u_{n}^{t_{n}} \text {. }
$$

(2) For every term $t$ in $\mathcal{L}_{1}(S)$ there exist constants $c_{1}, \ldots, c_{n}$ in $S$ and terms $t_{1}, \ldots, t_{n}$, which are either atoms or 1 , such that

$$
t \equiv c_{1} t_{1}+\cdots+c_{n} t_{n} .
$$

Proof. First suppose each $t_{j}$ in (1) is an atom. Then it is easy to rearrange and consolidate factors, obtaining an atom $t$ satisfying $t \equiv u_{1}^{t_{1}} \cdots u_{n}^{t_{n}}$. If each $t_{j}$ is an atom or 1 , then it is only necessary to let $c$ be the product of all $u_{j}$ which are constant and have $t_{j}$ equal to 1 . Again the remaining factors can be rearranged and consolidated to yield an atom $t$ which will satisfy $c \cdot t \equiv u_{1}^{t_{1}} \cdots u_{n}^{t_{n}}$.

EXAMPLE.

$$
\left(x^{2}\right)^{1} \cdot(3)^{x^{1}} \cdot(2)^{1} \cdot\left(x^{3}\right)^{1} \cdot(2)^{x^{1}} \cdot\left(x^{1}\right)^{x^{2}}=2 \cdot\left(x^{1}\right)^{x^{2}} \cdot(6)^{x^{1}} \cdot\left(x^{5}\right)^{1}
$$

Now suppose each of the terms $t_{j}$ can be represented as in (2). For example, consider $t_{1} \equiv c_{1} s_{1}+\cdots+c_{m} s_{m}$, where $c_{1}, \ldots, c_{m}$ are in $s$ and each $s_{j}$ is 1 or an atom. Then

$$
u_{1}^{t_{1}} \equiv\left(u_{1}^{c_{1}}\right)^{s_{1}} \cdots\left(u_{1}^{c_{m}}\right)^{s_{m}}
$$

and each $u_{1}^{c_{j}}$ is $\equiv$ to a constant $>1$ in $S$ or to $x^{a}$ for some $a \in S$. Then each factor $u_{j}^{t_{j}}$ reduces in this way, and we proceed as earlier.

Now we prove (2) by induction on the number of symbols in the terms being considered. Suppose $t$ and $s$ can be represented in the desired way:

$$
t \equiv c_{1} t_{1}+\cdots+c_{n} t_{n}, \quad s \equiv d_{1} s_{1}+\cdots+d_{m} s_{m},
$$

where each $c_{j}$ and $d_{i}$ is in $S$ and each $t_{j}$ and $s_{i}$ is an atom or 1 . Evidently $t+s$ is represented in the correct form. Also $t \cdot s$ is $\equiv$ to a sum of terms $c_{j} \cdot d_{i} \cdot t_{j} \cdot s_{i}$. Since each product of atoms is $\equiv$ to an atom (this is shown as part of the earlier argument), $t \cdot s$ is $\equiv$ to a term of the desired type. Finally, if $u$ is a constant $B>1$ in $S$ or a power $x^{a}$ for $a \in S$, then $u^{t}$ was shown earlier to have the desired form.

Theorem 1.10 (Normal Form TheOREM for $\varrho_{1}(S)$ ). For each term $t$ in $\varrho_{1}(S)$ there exist unique terms $t_{1}, \ldots, t_{n}$, each an atom or 1 , and unique constants $c_{1}, \ldots, c_{n}$ in $S$ such that $t_{n} \prec t_{n-1} \prec \cdots \prec t_{1}$ and $t \equiv c_{1} \cdot t_{1}+\cdots+c_{n} \cdot t_{n}$.

Proof. The existence of $c_{1}, \ldots, c_{n}$ and $t_{1}, \ldots, t_{n}$ follows from Proposition 1.9(2). (Rearrange the $t_{j}$ 's and consolidate to insure that $t_{n} \prec t_{n-1} \prec \cdots \prec t_{1}$.) Suppose $t$ is a term with two distinct representations of this type, say

$$
t \equiv c_{1} \cdot t_{1}+\cdots+c_{n} \cdot t_{n} \equiv d_{1} \cdot s_{1}+\cdots+d_{m} \cdot s_{m} .
$$

Without loss of generality we may assume $c_{1} \cdot t_{1}$ is distinct from $d_{1} \cdot s_{1}$.

Case 1. $t_{1}$ and $s_{1}$ are distinct. By Theorem 1.7(2) we have $t_{1} \neq s_{1}$, and we may thus assume $t_{1} \prec s_{1}$. By Theorem 1.7(1) there exists $c>0$ so that $x^{c} \cdot t_{1} \prec s_{1}$. But then for some constant $k>0$,

$$
t \equiv c_{1} \cdot t+\cdots+c_{n} \cdot t_{n} \preccurlyeq k \cdot t_{1} \prec d_{1} \cdot s_{1} \preccurlyeq d_{1} \cdot s_{1}+\cdots+d_{m} \cdot s_{m} \equiv t,
$$

which is impossible. 
Case 2. $t_{1}, s_{1}$ are identical and $c_{1} \neq d_{1}$. We may assume $c_{1}<d_{1}$. Using Theorem 1.7(1) we may obtain $c>0$ so that for each $j \geqslant 2, x^{c} \cdot t_{j} \prec t_{1}$. Take $\delta>0$ so that $c_{1}+\delta<d_{1}$. Then

$$
t \equiv c_{1} \cdot t_{1}+c_{2} \cdot t_{2}+\cdots+c_{n} \cdot t_{n} \prec c_{1} \cdot t_{1}+\delta \cdot t_{1} \preccurlyeq d_{1} \cdot s_{1} \preccurlyeq t,
$$

which is again a contradiction.

This completes the proof of the Normal Form Theorem.

We remark that in the case where $S$ equals $\mathbf{N}=\{1,2,3, \ldots\}$ this result gives a converse to an observation of Levitz [LEV]: this shows that every atom is an additive prime in his sense. For $\varrho_{1}(\mathbf{N})$, we have the same normal form representation as that given in [LEV], but here we show how to obtain the normal form rather explicitly. In $\S 4$, we will show that this procedure gives an algorithm which decides $t \preccurlyeq s$ for terms $t, s$ in $\mathcal{L}_{1}(\mathbf{N})$.

In the remainder of this section we draw some conclusions for terms in many variables that can be obtained from the results above for 1-variable terms, just by specializing variables in various ways.

Definition 1.11. (i) A tower in $\mathcal{L}(S)$ is any term of the form $u^{t}$, where $t \in \mathcal{L}(S)$ and $u$ is a constant $B>1$ in $S$ or $u$ is one of the variables $x_{j}$.

(ii) A tower $u^{t}$ is exponential if $t$ itself is a product of nonconstant towers.

(iii) A monomial in $\mathcal{L}(S)$ is a term of the form $a_{0} \cdot x_{i_{1}}^{a_{1}} \cdots x_{i_{k}}^{a_{k}}$ where $a_{0}$, $a_{1}, \ldots, a_{k} \in S$. (This includes constants as trivial monomials.)

We will be mainly concerned with products of towers (including the trivial product 1). Such a product will be called exponential if it is a nontrivial product of towers, each of which is exponential.

LEMMA 1.12. For each term t there exist monomial terms $M_{1}, \ldots, M_{k}$ and exponential products of towers $t_{1}, \ldots, t_{k}$ (including possibly 1 ), all in $\mathcal{L}(S)$, such that $t \equiv M_{1} \cdot t_{1}$ $+\cdots+M_{k} \cdot t_{k}$. Moreover, if $t$ is itself a product of towers, then we may take $k=1$ in this expression.

PROOF. This representation of $t$ is the result of a straightforward use of basic rules of algebra.

Now we consider "substitutions" which lead from $\mathcal{L}(S)$ to $\varrho_{1}(S)$. Let $t$ be a term in $\mathcal{L}(S)$ and suppose the variables in $t$ are among $x_{1}, \ldots, x_{n}$. Let $s_{1}, \ldots, s_{n}$ be terms in $\varrho_{1}(S)$, with each $s_{j}$ being either a constant $>1$ from $S$ or a power $x^{a}$ for $a \in S$. Let $t^{\prime}$ be the result of replacing $x_{j}$ in $t$ by $s_{j}(1 \leqslant j \leqslant n)$. Then $t^{\prime}$ is a term in $\varrho_{1}(S)$, as is easily proved by induction on the number of symbols in $t$.

We are particularly interested in the case where $s_{j}$ is $x$ (for some fixed $j$ ) and $s_{i}$ is a constant $a_{i}>1$ from $S$ for each $i \neq j(1 \leqslant i \leqslant n)$. In that case we will write the term $t^{\prime}$ as $t\left(\hat{a}_{j}\right)$.

Proposition 1.13. Let $t \in \mathcal{L}(S)$ be a product of towers, whose variables are among $x_{1}, \ldots, x_{n}$. Fix $j(1 \leqslant j \leqslant n)$ and let $a_{1}, \ldots, a_{j-1}, a_{j+1}, \ldots, a_{n}$ be constants. $>1$ from $S$. Then there exist a constant $c \in S$ and a term $s \in \mathcal{L}_{1}(S)$ which is either 1 or an atom such that $t\left(\hat{a}_{j}\right) \equiv c \cdot s$. 
Proof. By Proposition 1.9(1) it suffices to prove this when $t$ is a tower. In that case $t\left(\hat{a}_{j}\right)$ is of the form $v^{t^{\prime}\left(\hat{a}_{j}\right)}$ where $v$ is $x^{a}$ or a constant $>1$. The result follows using Proposition 1.9(1).

COROLlaRY 1.14. Let $t_{1}, t_{2}$ be products of towers in $\mathcal{L}(S)$ and let $a_{1}, \ldots, a_{j-1}$, $a_{j+1}, \ldots, a_{n}$ be constants $>1$ from $S$. Exactly one of the following conditions is true:

(1) For some $B>1$ and $a \in S, B^{x^{a}} \cdot t_{1}\left(\hat{a}_{j}\right) \preccurlyeq t_{1}\left(\hat{a}_{j}\right)$.

(2) For some $B>1$ and $a \in S, B^{x^{a}} \cdot t_{2}\left(\hat{a}_{j}\right) \preccurlyeq t_{1}\left(\hat{a}_{j}\right)$.

(3) For some $a, b, c, d, d \cdot x^{b} \cdot t_{1}\left(\hat{a}_{j}\right) \equiv c \cdot x^{a} \cdot t_{2}\left(\hat{a}_{j}\right)$. (Here $c, d$ are in $S$ and each of $a, b$ is either 0 or is in $S$.)

Note. Our main application of this is where $S=\{r \in \mathbf{R} \mid r \geqslant 1\}$; in that case the expression $B^{x^{a}}$ can be replaced by $B^{x}$ in clauses (1) and (2). Hence we have

COROllaRY 1.15 ( $B^{x}$ LEMma). Let $t_{1}, t_{2}$ be products of towers in $\varrho(S)$ where $S \subseteq \mathbf{R}^{\geqslant 1}=\{r \in \mathbf{R} \mid r \geqslant 1\}$. Let $a_{1}, \ldots, a_{j-1}, a_{j+1}, \ldots, a_{n}$ be constants $>1$ in $S$. Exactly one of the following conditions is true:

(1) For some $B>1, B^{x} \cdot t_{1}\left(\hat{a}_{j}\right) \preccurlyeq t_{2}\left(\hat{a}_{j}\right)$.

(2) For some $B>1, B^{x} \cdot t_{2}\left(\hat{a}_{j}\right) \preccurlyeq t_{1}\left(\hat{a}_{j}\right)$.

(3) For some $a, b, c, d$ (with $c, d \in S$ and $a, b$ either 0 or in $S$ ),

$$
d \cdot x^{b} \cdot t_{1}\left(\hat{a}_{j}\right) \equiv c \cdot x^{a} \cdot t_{2}\left(\hat{a}_{j}\right) .
$$

Proposition 1.16. If $t, s$ are products of towers, with both $t$ and $s$ being exponential, and if $M, N$ are monomials, then $M t \equiv N s \Rightarrow t \equiv s$.

Proof. Let the variables in $M, N, t, s$ be among $x_{1}, \ldots, x_{n}$. Fix $j(1 \leqslant j \leqslant n)$ and let $k$ be any integer $>1$. Define terms $t_{k}, s_{k}$ in $\varrho_{1}(S)$ by replacing every $x_{j}$ in $t, s$ by $k x$ and by replacing every $x_{i}(i \neq j)$ in $t, s$ by $x$. A straightforward induction argument shows that each $t_{k}, s_{k}$ is $\equiv$ to an exponential atom in $L_{1}(S)$. On the other hand, under the same substitutions $M$ and $N$ become $\equiv$ to monomials $M_{k} N_{k}$ in $\mathcal{E}_{1}(S)$ (i.e. terms of the form $a x^{b}$ ). If $M t \equiv N s$ then $M_{k} t_{k} \equiv N_{k} s_{k}$ for each $k$. Hence by the $B^{x}$ Lemma (Corollary 1.15) $t_{k} \equiv s_{k}$ for each integer $k \geqslant 1$ (and each choice of $\bar{j}=1,2, \ldots, n)$.

If $M$ is $c x_{1}^{a_{1}} \cdots x_{n}^{a_{n}}$ and $N$ is $d x_{1}^{h_{1}} \cdots x_{n}^{h_{n}}$, then this yields that for each $j=1,2, \ldots, n$ and each $k \geqslant 1$,

$$
c_{k}^{a} x^{A} \equiv d_{k}^{b} x^{B},
$$

where $A=\Sigma a_{j}$ and $B=\Sigma b_{j}$. It follows that $M$ and $N$ are identical and, hence, that $t \equiv s$, as desired.

COROLlary 1.17. Suppose $t, s$ are products of towers in $L(S)$. If there exist monomials $M, N$ (not necessarily in $\mathcal{L}(S)$ ) such that $M t \equiv N s$, then there is one product of towers $t_{1}$ in $\mathcal{L}(S)$ and monomials $M_{1}, N_{1}$ in $\mathcal{E}(S)$ such that $t_{1}$ is either 1 or an exponential product of towers and $t \equiv M_{1} t_{1}, s \equiv N_{1} t_{1}$.

Proof. By Lemma 1.12 there exist $t_{1}, s_{1}$ (which are each either 1 or an exponential product of towers) and monomials $M_{1}, N_{1}$ such that $t_{1}, s_{1}, M_{1}, N_{1}$ are all in $\mathcal{L}(S)$ and $t \equiv M_{1} t_{1}$ and $s \equiv N_{1} s_{1}$. Then $M M_{1} t_{1} \equiv N_{1} s_{1}$, so $t_{1} \equiv s_{1}$ by Proposition 1.16. 
2. Applying the Nevanlinna characteristic. In this section we will apply the methods and ideas of value distribution theory for functions defined by terms in the Levitz class $\varrho(S)$ (Definition 1.1). For technical reasons it seems necessary for us to assume that every constant $c$ in $S$ satisfies $c \geqslant 1$, and we make this assumption throughout this section and the next.

We first observe that all functions defined by terms in $\mathcal{L}(S)$ are analytic on the domain obtained by letting each variable range over the planar domain $\Omega=$ $\mathbf{C} \backslash(-\infty, 0]$ which is the complex plane minus an infinite slit along the negative real axis. (If $t \in \mathcal{L}(S)$ has variables among $x_{1}, \ldots, x_{n}$ then $t$ defines an analytic function on the domain $\Omega^{n}$ in complex $n$-space.)

Consider first the case $n=1$ : each $t \in \varrho_{1}(S)$ defines a function analytic on $\Omega$. This domain may be viewed as an angular region (of opening $2 \pi$ ), and Nevanlinna [NEV I] has defined and studied his characteristic in such regions. However his full analysis applies only for functions of finite order; so far as we know, no one has yet succeeded in proving the Lemma of the Logarithmic Derivative (LLD-see (C2.6)) in this context. The LLD is an essential tool for our purposes.

One could turn instead to the characteristic developed by Tsuji [TSU], and this might succeed. However, it is rather clumsy for making the kind of specific estimates we need (see especially Lemma 2.3).

Instead, we use the simple device of mapping the unit disc $\mathbf{D}=\{|z|<1\}$ onto $\Omega$ by an explicit mapping $\psi$. We identify a function $f$ in our class with the function $F=f \circ \psi$ on $\mathbf{D}$, and write $T_{\Omega}(r, f)=T_{\mathbf{D}}(r, F)$, where $T_{\mathbf{D}}$ is the usual Nevanlinna characteristic for the disc. (Later, we will drop the subscripts on the $T$ 's; the context will make it clear which domain is intended.) With this characteristic everything goes through smoothly.

The mapping $\psi(z)$ which maps the unit disc $\mathbf{D}$ conformally onto $\Omega$ is

$$
\psi(z)=\left(\frac{(1+z)}{(1-z)}\right)^{2}
$$

One possible difficulty in passing from $T_{\mathrm{D}}$ to $T_{\Omega}$ via a conformal mapping $\psi$ is that the derivatives of $F=f \circ \psi$ involve derivatives of $f$ combined with derivatives of $\psi$. In this case, however, $\psi$ and all its derivatives are of bounded characteristic, so this causes no trouble in the end.

In the case of several variables, $n \geqslant 2$, we work by just mapping the polydisc $\mathbf{D}^{n}=\left\{z=\left(z_{1}, \ldots, z_{n}\right) \in \mathbf{C}^{n}:\left|z_{j}\right|<1\right.$ for $\left.j=1,2, \ldots, n\right\}$ onto the product domain $\Omega^{n}$ on which our functions are defined. For this situation then, we need a characteristic for functions on the polydisc. There has been a rather extensive development of the Nevanlinna characteristic for several complex variables, but most of it uses an exhaustion of $\mathbf{C}^{n}$ by balls $\mathbf{B}_{r}^{n}=\left\{z=\left(z_{1}, \ldots, z_{n}\right) \in \mathbf{C}^{n}:\left|z_{1}\right|^{2}+\cdots+\left|z_{n}\right|^{2}<r^{2}\right\}$, $0<r<\infty$. Thus, also, the characteristic is developed for balls, while we require a Nevanlinna theory for polydiscs $\mathbf{D}_{r}^{n}=\left\{z=\left(z_{1}, \ldots, z_{n}\right) \in \mathbf{C}^{n}:\left|z_{1}\right|<r, \ldots,\left|z_{n}\right|<r\right\}$. (See [GAH] for a treatment of the characteristic based on balls.)

In response to a request from us, W. Stoll has kindly provided a detailed manuscript [STO] where the polydisc characteristic (complete with LLD) is worked 
out. (In particular, his paper has a detailed history of LLD in several complex variables, to which we refer the reader for the background of the subject. We merely mention here the book of Ronkin [RON] and the paper of Taylor [TAB] that give early mention of the characteristic for polydiscs, but do not do LLD.) We shall borrow heavily from this useful manuscript of Stoll, giving only enough of the definitions and results for the applications we have in mind, since the details are rather lengthy. The Stoll paper works out the theory from scratch, and is complete in itself.

Consider first the case of one variable, $n=1$. For meromorphic functions $f$ of one variable, defined on the unit disc $\mathbf{D}=\{z|| z \mid<1\}$, the characteristic function is defined for $0<r<1$ by

$$
T(r, f)=m(r, f)+N(r, f)
$$

this is a sum of two terms: the proximity function

$$
m(r, f)=\frac{1}{2 \pi} \int_{-\pi}^{\pi} \log ^{+}\left|f\left(r e^{i \theta}\right)\right| d \theta
$$

which measures how close, on the average, $f$ is to $\infty$, and the averaged counting function

$$
N(r, f)=\int_{0}^{r} \frac{n(t, f)}{t} d t,
$$

where $n(r)$ is the number of poles of $f$ in the disc $|z| \leqslant r$. Here, $r$ ranges over the interval $0<r<1$, and appropriate modifications must be made in the definitions of $N(r, f)$ if $f(0)=0$ or if $f(0)=\infty$. The function $\log ^{+}(t)$ is defined by setting $\log ^{+}(t)=\log (t)$ for $t \geqslant 1$ and $\log ^{+}(t)=0$ for $0 \leqslant t \leqslant 1$. The growth of the characteristic $T(r, f)$ as $r \rightarrow 1$ gives a very useful measure of the growth of $f$. The basic properties which we shall use are listed below.

(C2.0) $T(r, f)$ is a nondecreasing function of $r$ and a convex function of $\log r$.

(C2.1) $T(r, f+g) \leqslant T(r, f)+T(r, g)+O(1)$.

(C2.2) $T(r, f g) \leqslant T(r, f)+T(r, g)$.

(C2.3) $T(r, 1 /(f-a))=T(r, f)+O(1)$ for any complex constant $a$.

(C2.4) $T(r, f / g) \leqslant T(r, f)+T(r, g)+O(1)$.

(C2.5) $T\left(r, e^{g}\right) / T(r, g) \rightarrow \infty \quad$ as $r \rightarrow 1$ if $T(r, g)$ is unbounded.

Proofs of these results may be found in W. Hayman's book [HAY]. (C2.5) is a special case of a result of Clunie (see [HAY, p. 54]). The other basic fact we need about the characteristic $T(r, f)$ is the Lemma of the Logarithmic Derivative:

(C2.6) (LLD)

$$
m\left(r, f^{\prime} / f\right) \leqslant O\left(\log (T(r, f))+\log \frac{1}{1-r}\right),
$$

except possibly for $r$ lying in a set $E \subseteq(0,1)$ which is "thin" in the sense that $\int_{E} d t /(1-t) \leqslant 2$.

In particular, the above estimate on $m\left(r, f^{\prime} / f\right)$ holds for some $r$ in the interval $\rho<r<\rho^{\prime}$ provided that $r_{0}<\rho<1$ and $1-\rho^{\prime}<(1-\rho) / e^{2}$. 
When it comes to several variables the theory is substantially the same and the basic properties we need are still expressed in the same form (C2.0)-(C2.6). (See [STO] for the details of proofs.) For a meromorphic function $f\left(z_{1}, \ldots, z_{n}\right)$ defined on $\mathbf{D}^{n}$,

$$
m(r, f)=\frac{1}{(2 \pi)^{n}} \int_{-\pi}^{\pi} \cdots \int_{-\pi}^{\pi} \log ^{+}\left|f\left(r e^{i \phi_{1}}, \ldots, r e^{i \phi_{n}}\right)\right| d \phi_{1} \cdots d \phi_{n},
$$

where $0<r<1$. Also $N(r, f)$ is defined much as before, as an averaged counting function of the poles of $f$. Note that if $f$ is a holomorphic function on $\mathbf{D}^{n}$, then $T(r, f)=m(r, f)$.

(In [STO], a characteristic $T(\vec{r}, f)$ is developed for a vector variable $\vec{r}=\left(r_{1}, \ldots, r_{n}\right)$, but we use only the diagonal case $r_{1}=\cdots=r_{n}=r$.)

The basic properties above, including LLD, are shown to hold in [STO] or follow exactly as in the case of one variable (e.g. (C2.5)). One thing which needs explanation is the derivative $f^{\prime}$ that occurs in the LLD. When $n \geqslant 2$ we shall take $f^{\prime}$ to stand for the Euler operator

$$
f^{\prime}=D f=z_{1} \frac{\partial f}{\partial z_{1}}+\cdots+z_{n} \frac{\partial f}{\partial z_{n}} .
$$

This has the useful property that $D f=0$ if and only if $f$ is identically constant. (This is because $f$ may be expanded as a nicely converging sum of homogeneous polynomials and because $D P=m P$ for any homogeneous polynomial of degree $m$.)

Our basic tool is a lemma (proved in one dimension by Hiromi and Ozawa; see [HIO]) which we will state after a short discussion of it (Lemma 2.1). In essence, it goes back to the work of R. Nevanlinna [NEV II], and is used in studying linear relations between entire functions. The proof depends only on the properties we have listed of the characteristic function $T(r, f)$. In particular, it uses LLD. So now it carries over, without modification in the proof, to polydiscs. (We note that Brownawell [BRO] has a result in several variables that includes the $\mathrm{H}-\mathrm{O}$ Lemma, but his characteristic is for balls rather than for polydiscs, so we cannot use it here. We mention also the one-variable study by $\mathrm{H}$. Cartan [CAR] which obtains results similar to what can be proved using, say, the H-O Lemma. However, there does not seem to be agreement that the proofs in [CAR] are complete.)

We now state the polydisc version of the $\mathrm{H}-\mathrm{O}$ Lemma. As mentioned above, its proof is formally the same as the proof given in [HIO] for dimension 1, employing the polydisc version of the characteristic.

LEMMA 2.1 (H-O LEMMA). Let $a_{0}(z), \ldots, a_{n}(z)$ be meromorphic functions and let $g_{1}(z), \ldots, g_{n}(z)$ be holomorphic functions, defined on the domain $\mathbf{D}^{N}$. Suppose that these functions satisfy

$$
T\left(r, a_{j}\right)=o\left(\sum_{i=1}^{n} m\left(r, e^{g_{i}}\right)\right)
$$


for each $j=0,1, \ldots, n$;

$$
T\left(r, e^{g_{i}}\right) \neq O\left(\log \frac{1}{1-r}\right)
$$

for at least one $i=1,2, \ldots, n$. Under these hypotheses, if the identity

$$
\sum_{j=1}^{n} a_{j}(z) e^{g_{j}(z)}=a_{0}(z)
$$

holds for $z \in \mathbf{D}^{N}$, then there exists constants $c_{1}, \ldots, c_{n}$ (not all 0 ) so that

$$
\sum_{j=1}^{n} c_{j} \cdot a_{j}(z) e^{g_{j}(z)}=0
$$

for all $z \in \mathbf{D}^{N}$.

Our use of the Nevanlinna theory tools previously discussed comes entirely through this $\mathrm{H}-\mathrm{O}$ Lemma. Indeed, we use it only in cases where $g_{1}, \ldots, g_{n}$ are holomorphic functions, so that $T\left(r, e^{g_{t}}\right)=m\left(r, e^{g_{i}}\right)$ (and the prohibition that the function not take the value 0 at the origin is satisfied), and in cases where $a_{0}, a_{1}, \ldots, a_{n}$ are slowly growing functions. Because the $\mathrm{H}-\mathrm{O}$ Lemma plays such an important role in our approach, and because it is published in a relatively inaccessible place, we give here the short proof from [HIO]. We first note that on writing $f^{\prime}=\left(f^{\prime} / f\right) f$ we may use (LLD) and (C2.2) to estimate $T\left(r, f^{\prime}\right)$ when $f$ is holomorphic. We give our proof in the context of the plane $\mathbf{C}$. Minor modifications carry it over to the other domains we consider. Now let $G_{\nu}(z)$ be $a_{\nu}(z) e^{g_{\nu}(z)}$. Then we have

$$
\sum_{\nu=1}^{n} G_{\nu}(z)=a_{0}(z)
$$

By differentiating both sides of (i), we have

$$
\sum_{\nu=1}^{n} G_{\nu}^{(\mu)}(z)=a_{0}^{(\mu)}(z)
$$

which we rewrite as

$$
\sum_{\nu=1}^{n} G_{\nu}(z) \frac{G_{\nu}^{(\mu)}(z)}{G_{\nu}(z)}=a_{0}^{(\mu)}(z), \quad \mu=1, \ldots, m-1 .
$$

We regard this as a system of simultaneous linear equations in the $G_{\nu}$. Now we have

$$
G_{\nu}^{(\mu)}(z)=P_{\mu}\left(a_{\nu}, a_{\nu}^{\prime}, \ldots, a_{\nu}^{(\mu)}, g_{\nu}^{\prime}, \ldots, g_{\nu}^{(\mu)}\right) e^{g_{v}(z)}
$$

with a suitable polynomial $P_{\mu}$ of the indicated functions $a_{\nu}, a_{\nu}^{\prime}, \ldots, a_{\nu}^{(\mu)}, g_{\nu}^{\prime}, \ldots, g_{\nu}^{(\mu)}$. Thus we have

$$
T\left(r, \frac{G_{\nu}^{(\mu)}}{G_{\nu}}\right) \leqslant O\left(T\left(r, a_{\nu}\right)+T\left(r, g_{\nu}\right)\right)=o\left(\sum_{\nu=1}^{n} m\left(r, e^{g_{\nu}}\right)\right)
$$

outside a set of finite Lebesgue measure. Suppose, for the simultaneous equations (i) and (iii), the determinant $\Delta \neq 0$. By solving (iii) with respect to $G_{j}, j=1, \ldots, n$, we have, by Cramer's rule,

$$
G_{j}=\Delta_{j} / \Delta
$$


where

$$
\begin{aligned}
& \Delta=\left|\begin{array}{ccc}
1 & \ldots & 1 \\
G_{1}^{\prime} / G_{1} & \ldots & G_{n}^{\prime} / G_{n} \\
\cdots \ldots \ldots & \ldots & \ldots \\
G_{1}^{(n-1)} / G_{1} & \ldots & G_{n}^{(n-1)} / G_{n}
\end{array}\right| \\
& \Delta_{j}=\left|\begin{array}{ccccccc}
1 & \ldots & 1 & a_{0} & 1 & \ldots & 1 \\
\frac{G_{1}^{\prime}}{G_{1}} & & \frac{G_{j-1}^{\prime}}{G_{j-1}} & a_{0}^{\prime} & \frac{G_{j+1}^{\prime}}{G_{j+1}} & & \frac{G_{n}^{\prime}}{G_{n}} \\
\ldots & \ldots & \ldots & \ldots \ldots & \ldots & \ldots & \ldots \\
\frac{G_{1}^{(n-1)}}{G_{1}} & \ldots & \frac{G_{j-1}^{(n-1)}}{G_{j-1}} & a_{0}^{(n-1)} & \frac{G_{j+1}^{(n-1)}}{G_{j+1}} & \ldots & \frac{G_{n}^{(n-1)}}{G_{n}}
\end{array}\right| .
\end{aligned}
$$

Since

$$
T\left(r, \frac{G_{\nu}^{(\mu)}}{G_{\nu}}\right)=o\left(\sum_{\nu=1}^{n} m\left(r, e^{g_{v}}\right)\right)
$$

we have

$$
T(r, \Delta)=O\left(\sum_{\nu=1}^{n} m\left(r, e^{g_{\nu}}\right)\right), \quad T\left(r, \Delta_{j}\right)=o\left(\sum m\left(r, e^{g_{v}}\right)\right), \quad j=1, \ldots, n,
$$

outside a set of finite Lebesgue measure. Thus we have

$$
\begin{aligned}
m\left(r, e^{g_{\nu}}\right) & =T\left(r, e^{g_{\nu}}\right) \leqslant T\left(r, a_{\nu}\right)+T\left(r, G_{\nu}\right) \\
& \leqslant T\left(r, a_{\nu}\right)+T(r, \Delta)+T\left(r, \Delta_{\nu}\right)=o\left(\sum_{\nu=1}^{n} m\left(r, e^{g_{\nu}}\right)\right),
\end{aligned}
$$

and, hence,

$$
\sum_{\nu=1}^{n} m\left(r, e^{g_{\nu}}\right)=o\left(\sum_{\nu=1}^{n} m\left(r, e^{g_{\nu}}\right)\right)
$$

outside a set of finite Lebesgue measure. This is a contradiction. Consequently, the Wronskian $\Delta \equiv 0$ and the result follows.

In the context of $\mathbf{D}^{N}$ instead of $\mathbf{C}$, a few small changes must be made. First, $(\sharp)$ should be replaced by

$(\#)(\sharp) \quad T\left(r, \frac{G_{\nu}^{(\mu)}}{G_{\nu}}\right) \leqslant O\left(T\left(r, a_{\nu}\right)+T\left(r, g_{\nu}\right)+\log \frac{1}{1-r}\right)$

off the exceptional set, because we inherit the $\log (1 / 1-r)$ term from LLD. Then $(*)$ is to be replaced by

$$
T\left(r, \frac{G_{\nu}^{(\mu)}}{G_{\nu}}\right)=o\left(\sum_{\nu=1}^{n} m\left(r, e^{g_{\nu}}\right)\right)+O\left(\log \frac{1}{1-r}\right)
$$

off the exceptional set $E$. 
Now let $\rho_{n} \rightarrow 1^{-}$so that

$$
T\left(\rho_{n}, e^{g_{i}}\right) / \log \left(1-\rho_{n}\right)^{-1} \rightarrow+\infty .
$$

Then by LLD we can find $r_{n}$ outside the exceptional set $E$ such that

$$
1-\rho_{n}>1-r_{n}>\left(1-\rho_{n}\right) / 9 \text {. }
$$

It follows that $r_{n} \rightarrow 1$ and

$$
\log \frac{1}{1-r_{n}}=\log \frac{1}{1-\rho_{n}}+O(1)=o\left(T\left(\rho_{n}, e^{g_{i}}\right)\right)=o\left(T\left(r_{n}, e^{g_{i}}\right)\right) .
$$

Hence $m\left(r_{n}, e^{g_{i}}\right) / \log \left(1 /\left(1-r_{n}\right)\right) \rightarrow \infty$, and we reach, as before, the contradiction

$$
\sum_{\nu=1}^{n} m\left(r, e^{g_{\nu}}\right)=o\left(\sum_{\nu=1}^{n} m\left(r, e^{g_{\nu}}\right)\right),
$$

and the $\mathrm{H}-\mathrm{O}$ Lemma for $\mathbf{D}^{N}$ is proved.

The other specific way in which Nevanlinna theory comes into this paper (and the one thing which prevents us from taking a purely axiomatic approach using properties (C2.0)-(C2.6)) is in Lemma 2.3. Here we must make an estimate of the Nevanlinna characteristic of quotients of certain of the functions which are defined using the Levitz class terms we study here.

We now use the Nevanlinna theory concepts and methods discussed above to treat certain aspects of identities between terms in the Levitz class, especially those involving products of towers (see Definition 1.11).

The next result, the Small Characteristic Lemma (SCL), shows that only in trivial cases does the characteristic of a quotient of products of towers grow no more rapidly than $\log (1-r)^{-1}$. (This is the "dividing line" below which the Nevanlinna characteristic on the polydisc is not a useful tool.)

LEMMA 2.2 (SCL). If $Q$ is a quotient of products of towers in $\mathcal{L}(S)$ and if $T(r, Q)=O\left(\log (1-r)^{-1}\right)$, then there exist monomials $M_{1}, M_{2}$ so that $Q \equiv M_{1} / M_{2}$.

Note. We do not assert here that $M_{1}$ and $M_{2}$ are in $\mathcal{L}(S)$, but this can be deduced from Lemma 2.2 using Corollary 1.17, if desired.

Proof. Using the exponential form of Lemma 2.4, we reduce this result to a collection of 1-variable problems. These we handle using the $B^{x}$ Lemma from $\S 1$ (Corollary 1.15) together with the following standard estimate for the growth of functions defined on $\Omega^{n}$, transposed from the polydisc $\mathbf{D}^{n}$ using our conformal mapping $\psi(z)=((1+z) /(1-z))^{2}$.

LEMMA 2.3. Let $f$ be a holomorphic function defined on $\Omega^{n}$. If

$$
T(r, f)=O\left(\log (1-r)^{-1}\right),
$$

then there exists a positive constant $\alpha$ such that $f_{j}(x)=O\left(x^{\alpha \sqrt{x}}\right)$ for positive, real $x \rightarrow \infty$, where $f_{j}(x)=f\left(x_{1}, \ldots, x_{j-1}, x, x_{j+1}, \ldots, x_{n}\right)$ for fixed positive real values of $x_{1}, \ldots, x_{j-1}, x_{j+1}, \ldots, x_{n}$.

It is this estimate which entails our blanket assumption, made in this section and the next, that every constant in $S$ is $\geqslant 1$. 
Proof. We define $F\left(z_{1}, \ldots, z_{n}\right)$ for $z \in \mathbf{D}^{n}$ by

$$
F\left(z_{1}, \ldots, z_{n}\right)=f\left(\psi\left(z_{1}\right), \ldots, \psi\left(z_{n}\right)\right) ; \quad \psi(z)=\left(\frac{1+z}{1-z}\right)^{2} .
$$

Then $F$ is holomorphic on $\mathbf{D}^{n}$ and $T(r, F)=O\left(\log (1-r)^{-1}\right)$ as $r \rightarrow 1-$. (Recall that the characteristic of $f$ over $\Omega^{n}$ is defined to be the same as the characteristic of $F$ over $\mathbf{D}^{n}$.) Let $\beta>0$ be such that

$$
\int_{-\pi}^{\pi} \cdots \int_{-\pi}^{\pi} \log ^{+}\left|F\left(r e^{i \theta_{1}}, \ldots, r e^{i \theta_{n}}\right)\right| d \theta_{1} \cdots d \theta_{n} \leqslant \beta \log \left(\frac{1}{1-r}\right)
$$

as $r \rightarrow 1-$. Let $u=\log |F|$, so $u$ is a plurisubharmonic function on $\mathbf{D}^{n}$ and, hence, is $n$-subharmonic. (See [RUD, §3.2.1] for these concepts.) Hence, with $u^{+}=\max (u, 0)$, we have

$$
u\left(\tilde{x}_{1}, \ldots, \tilde{x}_{j-1}, \tilde{x}, \tilde{x}_{j+1}, \ldots, \tilde{x}_{n}\right) \leqslant \int u(\vec{\xi}) P(\vec{\xi}, \vec{z}) d \phi \leqslant \int u^{+}(\vec{\xi}) P(\vec{\xi}, \vec{z}) d \phi
$$

where

$$
\begin{aligned}
& \vec{\xi}=\left(\rho e^{i \phi_{1}}, \ldots, \rho e^{i \phi_{n}}\right), \\
& \vec{z}=\left(\tilde{x}_{1}, \ldots, \tilde{x}_{j-1}, \tilde{x}, \tilde{x}_{j+1}, \ldots, \tilde{x}_{n}\right), \quad d \phi=d \phi_{1} \cdots d \phi_{n},
\end{aligned}
$$

The integration is over $[-\pi, \pi]^{n}$, and $P(\vec{\xi}, \vec{z})$ is the Poisson kernel given by

$$
P(\vec{\xi}, \vec{z})=P\left(\vec{\xi}_{1}, z_{1}\right) P\left(\xi_{2}, z_{2}\right) \cdots P\left(\xi_{n}, z_{n}\right)
$$

(where $\vec{\xi}=\left(\xi_{1}, \ldots, \xi_{n}\right)$ and $\left.\vec{z}=\left(z_{1}, \ldots, z_{n}\right)\right)$, where

$$
P\left(\rho e^{i \phi}, r e^{i \theta}\right)=\frac{1}{2 \pi} \frac{\rho^{2}-r^{2}}{\rho^{2}-2 \rho r \cos (\theta-\phi)+r^{2}} \leqslant \frac{1}{2 \pi} \frac{\rho+r}{\rho-r} .
$$

Here, we choose $\rho$ so that $1 /(1-\rho)=2(1-\tilde{x})$. To complete the description of our notation, $x=\psi(\tilde{x})=((1+\tilde{x}) /(1-\tilde{x}))^{2}$, so $\tilde{x}=(\sqrt{x}-1) /(\sqrt{x}+1)$ and, hence, $1 /(1-\tilde{x})=(\sqrt{x}+1) / 2$, with corresponding notation for the subscripted $x$ 's and $\tilde{x}$ 's. Also, let $F_{j}(\tilde{x})=F\left(\tilde{x}_{1}, \ldots, \tilde{x}_{j-1}, \tilde{x}, \tilde{x}_{j+1}, \ldots, \tilde{x}_{n}\right)$ where $\tilde{x}_{i}$ is fixed for $i \neq j$. We choose $x$ so large that $1>\rho>\tilde{x}_{i}$ for $i \neq j$. As $x \rightarrow \infty, \rho \rightarrow 1-$ so that $\left(\rho+\tilde{x}_{j}\right) /\left(\rho-\tilde{x}_{j}\right)$ is bounded for $i \neq j, i \in\{1,2, \ldots, n\}$. Hence

$$
u(\tilde{x}) \leqslant \beta\left(\frac{\rho+\tilde{x}}{\rho-\tilde{x}}\right) \log \frac{1}{1-\rho}
$$

for a suitable constant $\beta$. It follows that

$$
u(\tilde{x}) \leqslant \frac{\gamma}{1-\tilde{x}} \log \frac{1}{1-\tilde{x}}
$$

for $\gamma$ chosen correctly, and thus

$$
\left|F_{j}(\tilde{x})\right| \leqslant \exp \left(\frac{\gamma}{1-\tilde{x}} \log \frac{1}{1-\tilde{x}}\right) \quad \text { as } \tilde{x} \rightarrow 1-.
$$

Now we pass back to $\Omega^{n}$ to get

$$
\left|f_{j}(x)\right|=\left|F_{j}(\tilde{x})\right| \leqslant \exp \left(\frac{\gamma}{1-\tilde{x}} \log \frac{1}{1-\tilde{x}}\right) \leqslant \exp (\alpha \sqrt{x} \log x)=x^{\alpha \sqrt{x}}
$$

for correctly chosen $\alpha>0$ as $x \rightarrow \infty$. This gives the result claimed in Lemma 2.3. 
Lemma 2.4 (AFFINE FORM). Let $n$ be an integer $\geqslant 2$ and let $L\left(t_{1}, \ldots, t_{n}\right)$ be a $C^{\infty}$ function defined on $(0, \infty)^{n}$. Suppose that for each $j=1,2, \ldots, n$ there exist functions $a_{j}$ and $b_{j}\left(\right.$ of $n-1$ variables $\left.\hat{t}_{j}=\left(t_{1}, \ldots, t_{j-1}, t_{j+1}, \ldots, t_{n}\right)\right)$ such that

$$
L\left(t_{1}, \ldots, t_{n}\right)=a_{j}\left(\hat{t}_{j}\right)+b_{j}\left(\hat{t}_{j}\right) \cdot t_{j}
$$

for all $\left(t_{1}, \ldots, t_{n}\right) \in(0, \infty)^{n}$.

Then there exist constants $\left\{C_{E} \mid E \subseteq\{1,2, \ldots, n\}\right\}$ such that

$$
L\left(t_{1}, \ldots, t_{n}\right)=\sum_{E} C_{E}\left(\prod_{j \in E} t_{j}\right) \quad \text { on }(0, \infty)^{n} .
$$

Proof. By induction on $n \geqslant 2$. Note first that the functions $a_{j}$ and $b_{j}$ are $C^{\infty}$ since $b_{j}=\partial L / \partial t_{j}$ and $a_{j}=L-b_{j} \cdot t_{j}$.

On differentiating $L$ with respect to $t_{i}$ we get (for any $j \neq i$ )

$$
b_{i}\left(\hat{t}_{i}\right)=\frac{\partial L}{\partial t_{i}}=\frac{\partial a_{j}\left(\hat{t}_{j}\right)}{\partial t_{i}}+\frac{\partial b_{j}\left(\hat{t}_{j}\right)}{\partial t_{i}} \cdot t_{j} .
$$

Differentiating this with respect to $t_{j}$ gives

$$
\frac{\partial b_{i}\left(\hat{t}_{i}\right)}{\partial t_{j}}=0+\frac{\partial b_{j}\left(\hat{t}_{j}\right)}{\partial t_{i}} \cdot 1 .
$$

Since the right side is clearly independent of $t_{j}$, we see that $b_{i}\left(\hat{t}_{i}\right)$ satisfies the hypotheses of Lemma 2.4 for each $i$. (That is, $b_{i}\left(\hat{t}_{i}\right)$ is an affine function of each of its variables taken separately.) Using this fact and equation (1), we see that $\partial a_{j}\left(\hat{t}_{j}\right) / \partial t_{i}$ is independent of $t_{i}$ for each $i \neq j$.

When $n=2$, this shows outright that $L$ has the correct form

$$
\begin{aligned}
L\left(t_{1}, t_{2}\right) & =a_{1}\left(t_{2}\right)+b_{1}\left(t_{2}\right) t_{1}=\left(\alpha+\beta t_{2}\right)+\left(\delta+\varepsilon t_{2}\right) t_{1} \\
& =\alpha+\beta t_{2}+\delta t_{1}+\varepsilon t_{1} t_{2} .
\end{aligned}
$$

When $n>2$, the induction hypothesis applied to $a_{1}, \ldots, a_{n}, b_{1}, \ldots, b_{n}$ and an easy calculation show that $L$ has the desired form.

Actually we will make use of the following exponential version of this fact.

LEMMA 2.4 (EXPONENTIAL Form). Let $n \geqslant 2$ and let $Q\left(x_{1}, \ldots, x_{n}\right)$ be a $C^{\infty}$ function defined on $(1, \infty)^{n}$ and taking values $>0$. Suppose that for each $j=1,2, \ldots, n$ there exist functions $A_{j}\left(\hat{x}_{j}\right)$ and $B_{j}\left(\hat{x}_{j}\right)$ such that

$$
Q\left(x_{1}, \ldots, x_{n}\right)=A_{j}\left(\hat{x}_{j}\right) x_{j}^{B_{j}\left(\hat{x}_{j}\right)}
$$

for all $\left(x_{1}, \ldots, x_{n}\right) \in(1, \infty)^{n}$.

Then there exist constants $\left\{C_{E} \mid E \subseteq\{1,2, \ldots, n\}\right\}$ such that

$$
Q\left(x_{1}, \ldots, x_{n}\right)=\exp \left(\sum_{E} C_{E}\left(\prod_{j \in E} \log \left(x_{j}\right)\right)\right)
$$

on $(1, \infty)^{n}$.

Proof. Change variables by setting $t_{j}=\log \left(x_{j}\right)(1 \leqslant j \leqslant n)$ and consider the function on $(0, \infty)^{n}$ defined by $L\left(t_{1}, \ldots, t_{n}\right)=\log Q\left(x_{1}, \ldots, x_{n}\right)$. It is immediate that 
$L$ satisfies the hypotheses of the Affine Form of Lemma 2.4, with $a_{j}=\log A_{j}$ and $b_{j}=B_{j}$. The conclusion of this result for $L$ gives the desired form for $Q=\exp (L)$.

Proof of LEMma 2.2 (SCL). Let $Q$ be the quotient of two products of towers which satisifes $T(r, Q)=O\left(\log (1-r)^{-1}\right)$. Note that it suffices to find monomials $M_{1}, M_{2}$ such that

$$
Q\left(x_{1}, \ldots, x_{n}\right)=M_{1}\left(x_{1}, \ldots, x_{n}\right) / M_{2}\left(x_{1}, \ldots, x_{n}\right)
$$

for all real $x_{1}, \ldots, x_{n}>1$. (Then apply the uniqueness of holomorphic functions and induction on the number of variables.) Thus we will consider $Q\left(x_{1}, \ldots, x_{n}\right)$ restricted to real values $>1$ of the variables $x_{1}, \ldots, x_{n}$.

Using the estimate in Lemma 2.3 we see that if we fix $x_{i}$ for $i \neq j$ and treat $Q$ as a function of $x_{j}$ only then

$$
Q\left(x_{1}, \ldots, x_{n}\right)=O\left(x_{j}^{\alpha_{j} \sqrt{x_{j}}}\right) .
$$

But now the $B^{x}$ Lemma (Corollary 1.15), applied to these functions of $x_{j}$, implies that there exist $A_{j}\left(\hat{x}_{j}\right)$ and $B_{j}\left(\hat{x}_{j}\right)$ so that

$$
Q\left(x_{1}, \ldots, x_{n}\right)=A_{j}\left(\hat{x}_{j}\right) x_{j}^{B_{j}\left(\hat{x}_{j}\right)} .
$$

Since this is true for each $j=1,2, \ldots, n$ and all $x_{1}, \ldots, x_{n}>1$, the Exponential Form of Lemma 2.4 yields constants $\left\{C_{E} \mid E \subseteq\{1,2, \ldots, n\}\right\}$ so that

$$
Q\left(x_{1}, \ldots, x_{n}\right)=\exp \left(\sum_{E} C_{E}\left(\prod_{j \in E} \log \left(x_{j}\right)\right)\right) .
$$

We need to show that $C_{E}=0$ unless $E$ is a singleton set. If this is not true, choose $E \subseteq\{1,2, \ldots, n\}$ of largest cardinality such that $C_{E} \neq 0$. Now set $x_{j}=x>1$ if $j \in E$ and set $x_{j}=2$ if $j \notin E$, for $1 \leqslant j \leqslant n$. Let $q(x)$ be the resulting function. It is clear that $q(x)$ is a constant times the quotient of a pair of atoms in $\varrho_{1}(S)$. But we then have a contradiction of the $B^{x}$ Lemma (Corollary 1.15), since $q(x)$ (or its reciprocal if $C_{E}<0$ ) satisfies an inequality of the form $A^{\log (x)^{p}} \leqslant q(x) \leqslant B^{\log (x)^{p}}$ for some $A, B>1$ and $p=\operatorname{card}(E)>1$. Hence $C_{E}$ must $=0$ unless $E$ is a singleton, so that $Q$ has the form

$$
Q\left(x_{1}, \ldots, x_{n}\right)=\exp \left(\sum_{j=1}^{n} c_{j} \log \left(x_{j}\right)\right)=x_{1}^{c_{1}} \cdots x_{n}^{c_{n}}
$$

as desired. (This is $\equiv$ to a quotient of monomials since some of the $c_{j}$ 's may be negative, while exponents in a monomial must be $\geqslant 0$.)

In the remainder of this section we prove two lemmas about products of towers which will be crucial in carrying out our inductive analysis of identities satisfied by general terms in the Levitz classes $\mathcal{L}(S)$ (see §3).

LEMMA 2.5 (FIRST POT LeMma). Let $T_{1}, \ldots, T_{m}$ be products of towers in $\mathcal{L}(S)$ and let $\pi_{j}$ be the function defined on $\Omega^{n}$ by $T_{j}$ for each $j=1,2, \ldots, n$. Suppose $p_{1}, \ldots, p_{m}$ are nontrivial polynomials in $x_{1}, \ldots, x_{n}$ and $\log \left(x_{1}\right), \ldots, \log \left(x_{n}\right)$. If the identity $p_{1} \pi_{1}+p_{2} \pi_{2}$ $+\cdots+p_{m} \pi_{m} \equiv 0$ holds on $\Omega^{n}$, then there exists a pair of distinct indices $i, j$ such that $\pi_{i} / \pi_{j}$ is $\equiv$ to a quotient of monomials on $\Omega^{n}$. 
Proof. We note that the coefficients in the polynomials $p_{j}$ are allowed to be arbitrary complex numbers.

The proof is by induction on $m$. We rewrite the identity as

$$
p_{1}\left(\pi_{1} / \pi_{m}\right)+\cdots+p_{m-1}\left(\pi_{m-1} / \pi_{m}\right)=-p_{m} .
$$

If for some $j(1 \leqslant j \leqslant m-1)$ we have $T\left(r, \pi_{j} / \pi_{m}\right)=O\left(\log (1-r)^{-1}\right)$, then we apply Lemma 2.2 (SCL) to get the desired result. Otherwise we may write $\pi_{j} / \pi_{m}=e^{g_{j}}$ for $1 \leqslant j \leqslant m-1$, and the $\mathrm{H}-\mathrm{O}$ Lemma (Lemma 2.1) applies. This yields constants $c_{1}, \ldots, c_{m-1}($ not all 0$)$ so that

$$
c_{1} p_{1}\left(\pi_{1} / \pi_{m}\right)+\cdots+c_{m-1} p_{m-1}\left(\pi_{m-1} / \pi_{m}\right)=0 .
$$

This yields an identity

$$
c_{1} p_{1} \pi_{1}+\cdots+c_{m-1} p_{m-1} \pi_{m-1} \equiv 0
$$

which has $\leqslant m-1$ terms. The induction hypothesis yields the desired result. (Note that the hypotheses of Lemma 2.6 are impossible if $m=1$. Hence the basis step $m=2$ must always be a case treated by application of Lemma 2.2 (SCL).)

LEMMA 2.6 (SECOND POT LEMMA). Let $T_{1}, \ldots, T_{m}$ be products of towers (including possibly 1) and let $\pi_{j}$ be the function defined on $\Omega^{n}$ by $T_{j}$ for each $j=1,2, \ldots, n$. Suppose $p_{1}, p_{2}, \ldots, p_{m}$ are nontrivial polynomials in $\log \left(x_{1}\right), \ldots, \log \left(x_{n}\right)$. If $\Sigma p_{j} \pi_{j} \equiv 0$, then for some $i \neq j, \pi_{k} / \pi_{j}$ is identically constant.

Proof. Using the first POT Lemma and Corollary 1.17 (possibly a number of times) there must exist monomials $M_{1}, \ldots, M_{m}$ and a product of towers $t$ such that $T_{j} \equiv M_{j} t$ holds for each $j=1,2, \ldots, n$. Then $\Sigma p_{j} M_{j} \equiv 0$. Since $\Sigma p_{j} M_{j}$ is a polynomial in $x_{1}, \ldots, x_{n}$ and $\log \left(x_{1}\right), \ldots, \log \left(x_{n}\right)$ and since $p_{1}, \ldots, p_{m}$ are nontrivial, it follows by an elementary argument that there must exist $i \neq j$ such that $M_{i} / M_{j}$ is identically constant. This implies

$$
\pi_{i} / \pi_{j} \equiv M_{i} t / M_{j} t \equiv M_{i} / M_{j} \equiv \text { constant }
$$

completing the proof.

3. A Normal Form Theorem for $\mathcal{L}(S)$ and completeness theorems for some systems of identities. In this section we prove a Normal Form Theorem for terms, in many variables, from the Levitz class $\mathcal{L}(S)$. From this will follow a positive solution to Tarski's High School Algebra Conjecture for terms in $\varrho(S)$, as well as in some other special cases which we treat briskly.

Definition 3.1. We define for $t \in \mathcal{L}(S)$, the concept $t$ is an atom by induction on the number of symbols in the formal term $t$ :

$t$ is an atom if it is of the form $u_{1}^{t_{1}} \cdots u_{m}^{t_{m}}(m \geqslant 1)$, where:

(i) each $u_{j}$ is a constant $B>1$ from $S$ or a power of a variable $x_{i}^{a}$ where $a \in S$;

(ii) each $t_{j}$ is an atom or 1 ;

(iii) if $u_{j}$ is constant then $t_{j}$ is not 1 ;

(iv) if $u_{i}, u_{j}$ are both constant and $i \neq j$, then $t_{i}, t_{j}$ are not identical terms;

(v) if $u_{i}, u_{j}$ are powers of the same variable $x_{k}$ and $i \neq j$, then $t_{i}, t_{j}$ are not identical terms;

(vi) for each $i<j$, the term $u_{i}^{t_{i}}$ is alphabetically earlier than $u_{j}^{t_{j}}$. 
Note. The alphabetical order mentioned in (vi) comes from an assumed linear ordering on the symbols used in constructing terms in $\varrho(S)$, including the constants from $S$. This gives a linear ordering on $\mathcal{L}(S)$ which we use, here and below, to resolve ambiguity in the order of terms in a product or a sum. Any linear ordering on $\mathcal{E}(S)$ would do as well as this one.

It should be noted than an atom in $\mathcal{L}(S)$, according to Definition 3.1, which contains only the variable $x_{1}$ (and is hence a term in $\mathcal{L}_{1}(S)$ ) need not be an atom in the sense of Definition 1.3. This is because we used a different linear ordering (the eventual dominance ordering) to resolve ambiguities in the order of factors in that earlier definition. In any case, these are technical notions and no confusion should arise from this double use of one word. Moreover, if $t \in E_{1}(S)$ and $t$ is an atom in the sense of Definition 3.1, then there exists $s \in E_{1}(S)$ such that $t \equiv s$ and $s$ is an atom in the sense of Definition 1.3. Indeed, $s$ is obtained from $t$ by a series of rearrangements of factors in products that appear within $t$.

Note also that each atom in $E(S)$ is a product of towers.

DEFINITION 3.2. A normal form term in $E(S)$ is a term of the form $c_{1} t_{1}+\cdots+c_{k} t_{k}$, where each $t_{j}$ is an atom or 1 , each $c_{j}$ is a constant in $S$ and for each $i<j, t_{i}$ is alphabetically (strictly) earlier than $t_{j}$.

Our next result asserts that each term $t$ in $\varrho(S)$ is $\equiv$ to a normal form term and that such a term can be obtained from $t$ by "elementary algebraic manipulations". We express this in terms of provability from the elementary High School Axioms, together with the arithmetic of numbers in $S$.

By the High School Axioms we mean the following:

$$
\begin{aligned}
x+(y+z) & =(x+y)+z, & x^{y+z} & =x^{y} \cdot x^{z}, \\
x+y & =y+x, & x^{y} & =\left(x^{y}\right)^{z}, \\
x(y z) & =(x y) x, & (x y)^{z} & =x^{z} \cdot y^{z}, \\
x y & =y x, & x^{1} & =x . \\
x(y+z) & =x y+x z, & 1^{x} & =1 . \\
1 \cdot x & =x, & & x y
\end{aligned}
$$

By the arithmetic of numbers in $S$ we mean the set of all possible numerical axioms such as $c_{1}+c_{2}=c_{3}$, where $c_{3}$ is a constant representing the sum of the numbers (in $S$ ) represented by the constants $c_{1}, c_{2}$. Not only axioms involving addition, but also multiplication and exponentiation need to be included.

If $t, s \in \mathcal{L}(S)$, then we write $\vdash_{s} t=s$ to mean there is a formal deduction of the identity $t=s$ from the High School Axioms and the axioms for arithmetic in $S$, described above.

Evidently $\vdash_{s} t=s$ implies $t \equiv s$, since the axioms are true identities and the rules of inference preserve truth. Tarski's Conjecture is that the converse implication is also true.

LeMma 3.3. For any term $t$ in $\mathcal{L}(S)$ there is a normal form term $s$ in $\mathcal{L}(S)$ so that $\mathrm{r}_{\mathrm{S}} t=s$.

Proof. By a straightforward induction on the number of symbols in $t$. The High School Axioms allow for the usual algebraic manipulations and this is all that is required to put $t$ into normal form. 
THEOREM 3.4 (NORMAL FORM THEOREM FOR $\mathcal{L}(S)$ ). If $t$, $s$ are normal form terms in $\ell(S)$ and $t \equiv s$, then $t, s$ are identical terms.

Proof. We prove a stronger statement: Suppose $p_{1}, \ldots, p_{m}(m \geqslant 1)$ are nontrivial polynomials in $\log \left(x_{1}\right), \ldots, \log \left(x_{n}\right)$, with arbitrary coefficients, and $t_{1}, \ldots, t_{m}$ are distinct terms in $\mathcal{L}(S)$, each either 1 or an atom. Then $p_{1} t_{1}+\cdots+p_{m} t_{m} \neq 0$. We prove this by induction on the total number of symbols in the sequence $t_{1}, \ldots, t_{m}$.

It is clear that this must be true when $m=1$, so suppose $m>1$. By the Second POT Lemma (Lemma 2.6) there exist $i \neq j$ and a constant $c$ so that $t_{i} \equiv c t_{j}$. We first note that $c$ must equal 1: the results of replacing each variable $x_{k}$ by $x$ in $t_{i}$ and $t_{j}$ are $\equiv$ to atoms in $\mathcal{L}_{1}(S)$ (in the sense of Definition 1.3) or to 1 , as discussed above. Hence Theorem 1.7 implies $c=1$. Clearly we may suppose $t_{i}, t_{j}$ are atoms.

Suppose $t_{i}$ is $u_{1}^{r_{1}} \cdots u_{k}^{r_{k}}$ and $t_{j}$ is $v_{1}^{s_{1}} \cdots v_{l}^{s_{l}}$, satisfying the restrictions in Definition 3.1. We have $t_{i} \equiv t_{j}$ so that on taking logarithms we get

$$
\log \left(u_{1}\right) \cdot r_{1}+\cdots+\log \left(u_{k}\right) \cdot r_{k} \equiv \log \left(v_{1}\right) \cdot s_{1}+\cdots+\log \left(v_{l}\right) \cdot s_{l} .
$$

This is almost in a form to which the induction hypothesis can be applied. Certainly the sequence of terms $r_{1}, \ldots, r_{k}, s_{1}, \ldots, s_{l}$ has strictly fewer symbols than does $t_{1}, \ldots, t_{m}$. Let $t_{1}^{\prime}, \ldots, t_{m}^{\prime}$ be a list of the distinct terms among $r_{1}, \ldots, r_{k}, s_{1}, \ldots, s_{l}$. For each $1 \leqslant i \leqslant h$ let $p_{i}$ be the sum of terms $\log \left(u_{j}\right)$ for which $r_{j}$ equals $t_{i}^{\prime}$ and let $q_{i}$ be the sum of terms $\log \left(v_{j}\right)$ for which $s_{j}$ equals $t_{i}^{\prime}$. Then by rearranging terms in the identity above, we get

$$
p_{1} t_{1}^{\prime}+\cdots+p_{h} t_{h}^{\prime} \equiv q_{1} t_{1}^{\prime}+\cdots+q_{h} t_{h}^{\prime} .
$$

The induction hypothesis yields that for each $1 \leqslant i \leqslant h$, the functions $p_{i}$ and $q_{i}$ must be identically equal.

We now may use the restrictions on $t_{i}$ and $t_{j}$ which are given in Definition 3.1, since we know $t_{i}$ and $t_{j}$ are atoms. We will show that $t_{j}$ and $t_{j}$ are identical terms. Each base $u_{\alpha}$ which appears in $t_{i}(1 \leqslant \alpha \leqslant k)$ is either a constant $B>1$ from $S$ or it is a power $x_{\beta}^{a}$ of one of the variables, with exponent $a \in S$. Moreover, each particular exponent can occur at most once with a constant base and at most once with a base involving each particular variable $x_{\beta}$. Also the exponent 1 may not occur with a constant base. This means that each $p_{\alpha}(1 \leqslant \alpha \leqslant h)$ will be, in the form we defined it, a sum of at most $n+1$ terms, including at most once the log of a constant and at most once for each variable $x_{\beta}$ an expression $\log \left(x_{\beta}^{a}\right)$. It follows that from $t_{1}^{\prime}, \ldots, t_{h}^{\prime}$ and from $p_{1}, \ldots, p_{h}$ we can recover the exact form of $t_{i}$.

The same remarks apply to $t_{j}$ and the functions $q_{1}, \ldots, q_{h}$. Thus it follows that $t_{i}$ and $t_{j}$ must be identical. But this contradicts the original hypotheses, so the theorem is proved.

Corollary 3.5 (TARSki's Conjecture for the Levitz Class $\mathcal{L}(S)$ ). Let $S \subseteq\{r$ $\in \mathbf{R} \mid r \geqslant 1\}$ be closed under addition, multiplication and (2-variable) exponentiation. If $t, s$ are any two terms in $\mathcal{L}(S)$ and if $t \equiv s$, then $\vdash_{S} t=s$. That is, the identity $t=s$ is provable from the High School Axioms together with axioms giving the addition, multiplication and exponentiation facts for $S$. 
Proof. Given $t$ and $s$, by Lemma 3.3 there are normal form terms $t_{1}, s_{1}$ so that $\vdash_{s} t=t_{1}$ and $\vdash_{S} s=s_{1}$. If $t \equiv s$ then also $t_{1} \equiv s_{1}$, from which it follows that $t_{1}, s_{1}$ are identical, by the Normal Form Theorem. Hence $\vdash_{s} t=s$.

REMARK. Strictly speaking, the proof given does not cover the case $S=\varnothing$ in Corollary 3.5 , since we have been making the blanket assumption throughout that $S \supseteq \mathbf{N}=\{1,2,3, \ldots\}$. However Tarski's Conjecture for $\mathscr{L}(\varnothing)$ is true. Indeed, if $t \equiv s$ and $t, s$ are Levitz class terms which contain no constants, then $t=s$ is provable using only those axioms which mention no constants. (I.e., the axioms for 1 are not needed.) To prove this one simply retraces our whole argument, but omitting all constants.

In the remainder of this section we use the Normal Form Theorem to prove completeness results, similar to Corollary 3.5, in two other situations. In the first of these, we consider terms which contain only exponentiation, giving a new proof of a result first proved in the Ph.D. thesis of A. Tarski's student Charles Martin [MAR], but unfortunately never published. In the second application we consider terms which contain only exponentiation and multiplication.

DEFINITION 3.6. Let $S$ be a set of real numbers $\geqslant 1$, closed under (2-variable) exponentiation, $a^{b} . \mathcal{E}(S)$ is the smallest class of terms that contains each variable $x_{1}$, $x_{2}, \ldots$ and each constant $c \in S$ and which contains $s^{t}$ for each $s, t \in \mathcal{E}(S)$.

Note that $\mathcal{E}(S)$ contains many terms, such as $\left(x^{x}\right)^{x}$ which are not in any Levitz class. However, we can define a mapping $\#$ from $\mathcal{E}(S)$ into $\mathcal{L}(S)$ in a natural way, which allows us to make use of our results about $\mathcal{L}(S)$. (For example the term above is mapped to $x^{x \cdot x}$.)

For $t \in \mathcal{E}(S)$ we define $t^{\#} \in \mathcal{L}(S)$ by induction on the number of symbols in $t$. If $t$ is a variable or a constant, then $t^{\#}$ is the same as $t$. Otherwise there is a variable or constant $u_{0}$ and terms $u_{1}, u_{2}, \ldots, u_{n}$ in $\mathcal{E}(S)(n \geqslant 1)$ so that $t$ is the iterated exponential

$$
\left(\cdots\left(u_{0}^{u_{1}}\right)^{u_{2}} \cdots\right)^{u_{n}}
$$

If $u_{0}$ is 1 , then we define $t^{\#}$ to be 1 also. Otherwise we construct $u_{1}^{\#}, \ldots, u_{n}^{\#}$ and define $t^{\sharp}$ to be the unique term in $\mathcal{L}(S)$ which is $\equiv$ to the term $u_{0}^{u^{\sharp} \cdot u^{\#} \cdots u_{n}^{\sharp}}$ and which is an atom in $\mathcal{L}(S)$. We omit the detailed proof that such an atom exists. However, it is useful to note that each $u_{j}^{\#}(1 \leqslant j \leqslant n)$ is a constant or an atom. The term $t^{\#}$ is constructed by taking its base to be $u_{0}$ raised to a power which is the product of the constant $u_{j}^{\#}$ terms. The exponent of $t^{\#}$ is 1 or an atom which is formed from the product of the $u_{j}^{\#}$ terms which are atoms. Construction of this exponent involves only consolidation of like terms and reordering of factors in the product.

COROLlaRY 3.7. If $t$, $s$ are terms in $\mathcal{E}(S)$ and $t \equiv s$, then there is a formal proof of the identity $t=s$ from the exponential axioms $1^{x}=1$ and $\left(x^{y}\right)^{z}=\left(x^{z}\right)^{y}$ together with all axioms giving the facts of exponentiation in $S$. (That is, all numerical axioms $c_{1}^{c_{2}}=c_{3}$ which represent true facts about $S$.)

Proof (Sketch). For each $t \in \mathcal{E}(S)$ the term $t^{\#} \in \mathcal{L}(S)$ defined above is an atom and $t \equiv t^{\sharp}$. If $t, s \in \mathcal{E}(S)$ and $t \equiv s$, then $t^{\#}$ and $s^{\#}$ are identical by the Normal 
Form Theorem for $\mathcal{L}(S)$. From this it follows that $t=s$ can be proved using the rearrangements allowed by the permitted axioms. This is because the rearrangements used in forming $t^{\#}$ from $t$ only involve the consolidation or reordering of the iterated exponents in $t$. This is precisely what is allowed for using the exponential axiom $\left(x^{v}\right)^{z}=\left(x^{z}\right)^{y}$. We omit the details.

The result proved by Charles Martin in his thesis [MAR] is not exactly contained in Corollary 3.7-it corresponds to taking $S$ to be empty and then using only the axiom $\left(x^{y}\right)^{z}=\left(x^{z}\right)^{y}$-but it can be proved by exactly the same method. The argument in Martin's thesis uses a proof-theoretic analysis. (See also [TAW] for some discussion of the many interesting results in this thesis.)

Next we briefly consider terms which are constructed using only exponentiation and multiplication.

Definition 3.8. Let $S$ be a set of real numbers $\geqslant 1$, closed under (2-variable) exponentiation $x^{y}$ and under multiplication. $\mathscr{E} \mathfrak{M}(S)$ is the smallest class of terms that contains each variable $x_{1}, x_{2}, \ldots$ and each constant $c \in S$ and which contains $s^{t}$ and $s \cdot t$ for each $s, t \in \mathcal{E} \Re(S)$.

COROLlaRY 3.9. If $t$, $s$ are terms in $\mathscr{G} \Re(S)$ and $t \equiv s$, then there is a formal proof of the identity $t=s$ from the axioms

$$
\begin{array}{cc}
x y=y x, & x^{z} \cdot y^{z}=(x y)^{z}, \\
x(y z)=(x y) z, & \left(x^{y}\right)^{z}=x^{y z}, \\
1 \cdot x=x, & 1^{x}=1,
\end{array}
$$

together with all axioms giving the facts of exponentiation and of multiplication in $S$. (If $S=\varnothing$ then only the 4 axioms which contain no constants are needed.)

Proof (Sketch). The basic idea is the same as in the proof of Corollary 3.7. For each $t \in \mathcal{E} \Re(S)$, one defines a term $t^{\#}$ which is a constant times an atom in $\mathcal{L}(S)$. Moreover, in this setting $t^{\#}$ is also a term in $\mathscr{E} \mathscr{N}(S)$ and the equality $t=t^{\#}$ is provable using only the axioms listed in the statement of Corollary 3.9. In part the construction of $t^{\#}$ (as a term in $\mathcal{L}(S)$ not containing any addition symbols) follows by repeated use of the identities $(u \cdot v)^{w}=u^{w} \cdot v^{w},\left(u^{v}\right)^{w}=u^{v \cdot w}$ to simplify the base of each exponential. The other part is essentially like Lemma 3.3: one needs to show that any term in $\mathcal{E}(S)$ which contains no + is provably equal to a constant times an atom, with the proof using only the axioms listed earlier (which are precisely the HS axioms not containing any + ).

Now suppose $t, s \in \mathcal{E} \mathscr{N}(S)$ and $t \equiv s$. Then $t^{\sharp} \equiv s^{\#}$, so that $t^{\sharp}$ and $s^{\#}$ are identical, by the Normal Form Theorem. Hence $t=s$ is provable in the desired way.

Note. The observation that underlies the previous proof shows that $\mathscr{L}(S)$ is "essentially" closed under exponentiation $s^{t}$, where $t$ is any term in $\varrho(S)$ and $s$ is any term which contains no + .

4. Decidability of $\preccurlyeq$ on $\mathcal{L}_{1}(\mathbf{N})$. Levitz [LEV] gave a mathematical analysis of the eventual dominance relation $\preccurlyeq$ on $\mathcal{L}_{1}(\mathbf{N})$, showing that the order type of $\preccurlyeq$ on this set is $\varepsilon_{0}$. However, he did not give an algorithm for deciding the relation $t \preccurlyeq s$ for $t, s \in \mathcal{L}_{1}(\mathbf{N})$. We give such an algorithm here. 
Proposition 4.1. (A) There is an algorithm which decides whether or not t (a term in $\mathrm{L}_{1}(\mathbf{N})$ ) is an atom (in the sense of Definition 1.3) or 1 .

(B) There is an algorithm which decides $t \preccurlyeq s$ when $t$ and $s$ are atoms in $\mathcal{L}_{1}(\mathbf{N})$.

Proof. These algorithms are defined simultaneously. They operate by recursion on the number of symbols in their inputs.

(A) Give $t$, we can effectively recognize if it is in the form $u_{1}^{t_{1}} \cdots u_{n}^{t_{n}}$ where $u_{1}, \ldots, u_{n}$ are constants or powers of $x$ and $t_{1}, \ldots, t_{n}$ are terms. If so, we can apply algorithm (A) recusively to decide whether or not $t_{1}, \ldots, t_{n}$ are atoms or 1 . We can apply algorithm (B) recursively to determine the $\preccurlyeq$ ordering of $t_{1}, \ldots, t_{n}$. Then we can apply Theorem 1.7 to decide whether or not $u_{n}^{t_{n}} \preccurlyeq \ldots \preccurlyeq u_{1}^{t_{1}}$. Finally the rest of the conditions in the definition of atom are easy to check effectively (see Definition 1.3).

(B) Given atoms $t, s$, we may effectively find $t_{1}, \ldots, t_{n}, u_{1}, \ldots, u_{n}, s_{1}, \ldots, s_{m}$, $v_{1}, \ldots, v_{m}$ such that $t$ is $u_{1}^{t_{1}} \cdots u_{n}^{t_{n}}$ and $s$ is $v_{1}^{s_{1}} \cdots v_{m}^{s_{m}}$. Then we can apply algorithm (B) recursively to decide the $\preccurlyeq$ ordering among $t_{1}, \ldots, t_{n}, s_{1}, \ldots, s_{m}$. This plus Theorem 1.7 give a decision as to whether or not $t \preccurlyeq s$ holds.

The next result asserts there is an algorithm which yields, for each term $t$ in $\varrho_{1}(S)$, its normal form representation. (The same is true for many-variable terms in $\mathcal{L}(S)$, but we will not use that result here.)

Proposition 4.2. There is an algorithm which yields, for each $t \in \mathcal{E}_{1}(\mathbf{N})$, a sequence $t_{1}, \ldots, t_{n}$ which are atoms or 1 , such that $t_{n} \preccurlyeq \cdots \preccurlyeq t_{1}$ and $t \equiv t_{1}+\cdots+t_{n}$.

Proof. This algorithm uses a combination of algebraic manipulations (repeatedly writing terms as sums of products of towers) together with use of the algorithms of Proposition 4.1, used to insure that each product of towers has its factors written in the correct $\preccurlyeq$ order.

REMARK. This representation of $t$ as a sum of atoms and 1's is (by Theorem 1.7) the same as Levitz' Additive Normal Form for $t$. The added information here is that we can identify the "additive primes" $[\mathbf{L E V}]$ (which are shown by Theorem 1.7 to be the same as the "atoms" of Definition 1.3) in a simple effective way.

This, together with Theorem 3.3 of [LEV], give immediately the desired algorithm for $\preccurlyeq$ on $\mathcal{L}_{1}(\mathbf{N})$.

THEOREM 4.3. There is an algorithm which decides $\preccurlyeq$ when $t$, $s$ are arbitrary terms in $\varrho_{1}(\mathbf{N})$.

Proof. Using the algorithm of Proposition 4.2, write $t \equiv t_{1}+\cdots+t_{n}$ and $s \equiv s_{1}$ $+\cdots+s_{m}$ where $t_{1}, \ldots, t_{n}, s_{1}, \ldots, s_{m}$ are atoms or 1's and $t_{n} \preccurlyeq \cdots \preccurlyeq t_{1}, s_{m} \preccurlyeq \cdots \preccurlyeq$ $s_{1}$. Find the smallest $i \leqslant \min (m, n)$ for which $t_{i} Z s_{i}$. If no such $i$ exists, then $t \preccurlyeq s$ holds iff $n \leqslant m$. If such an $i$ exists, then $t \leqslant s$ holds if $t_{i} \preccurlyeq s_{i}$ (by Theorem 3.3 of [LEV]).

Note. The same argument yields an algorithm which decides $\preccurlyeq$ on $\mathcal{L}_{1}(S)$, relative to an oracle which computes addition, multiplication and exponentiation on $S$ and which decides $\leqslant$ on $S$. (Here $S$ is any effectively given countable subset of $\mathbf{R}^{+}$which is closed under,$+ \cdot$ and 2-variable exponentiation $a^{b}$.) 
5. Exponential terms with constant bases, over the complex numbers. Here we consider expressions that are built up from variables and complex constants using addition, multiplication and the 1-variable exponential function $e^{x}$ (where $e$ is the usual base of the natural logarithm). We prove a version of Tarski's High School Algebra Conjecture for these expressions. (This result was proved independently by L. van den Dries [VDD] and, for terms containing just one variable, by A. Wilkie [WIL I]. Their methods are quite different from ours.) We also settle positively a conjecture, due to $\mathrm{S}$. Schanuel, which asserts that if $f$ is a function on $\mathbf{C}^{n}$ which is defined by an expression of this type and if $f$ is nowhere equal to 0 , then $f=e^{g}$ for a function $\mathbf{g}$ on $\mathbf{C}^{n}$ which is also defined by an expression of the kind considered here.

DEFinition 5.1. $\Sigma$ is the smallest class of terms which contains the variables $x_{1}$, $x_{2}, \ldots$ and a constant for each complex number, and which contains the terms $s+t$, $s \cdot t$ and $\exp (t)$ for each $s, t \in \Sigma$.

Here we interpret $\exp (t)$ to stand for $e^{t}$. We note that if $t \in \Sigma$ and the variables of $t$ are among $x_{1}, \ldots, x_{\mathrm{n}}$, then $t$ defines a holomorphic function on all of $\mathbf{C}^{n}$. As before, if $s \in \Sigma$ also has its variables among $x_{1}, \ldots, x_{n}$, we write $t \equiv s$ to mean that $t$ and $s$ define the same function on $\mathbf{C}^{n}$. (Various equivalent formulations of this definition are possible in special cases because of the uniqueness of holomorphic functions. For example, if $t$ and $s$ contain only real constants, we may be interested only in the functions they define on $\mathbf{R}^{n}$. But $t \equiv s$ will hold as long as $t$ and $s$ define the same function on $\mathbf{R}^{n}$, or even on $S^{n}$ where $S \subseteq \mathbf{C}$ is any set with a limit point in $\mathbf{C}$.)

In this section we will use Nevanlinna theory for functions defined on $\mathbf{C}^{n}$ $(n=1,2, \ldots)$ in a way similar to its use in $\$ 2$ on the polydisc $\mathbf{D}^{n}$ (and the conformally equivalent domain $\Omega^{n}$ ). Here we may either use a characteristic based on polydiscs (as was done above) or based on an exhaustion of $\mathbf{C}^{n}$ by balls. On $\mathbf{C}^{n}$ the LLD has been proved for both types of characteristic-for the characteristic based on balls this was done by Vitter [VIT]. (See also [GAH].)

In either case the properties corresponding to $(\mathrm{C} 2.0)-(\mathrm{C} 2.6)$ are true and, hence, the $\mathrm{C}^{n}$ version of the $\mathrm{H}-\mathrm{O}$ Lemma (Lemma 2.1) is also true. (A few small changes of interpretation are needed, of course. For example, in (C2.5) and in the "big- $O$ " and "little- $o$ " notations, one replaces $r \rightarrow 1$ by $r \rightarrow \infty$. Also in the LLD (C2.6) the extra term is $\log (r)$ instead of $\log (1-r)^{-1}$. Here the exceptional set $E$ is "thin" in the sense that it has finite Lebesgue measure $\left(E \subseteq(0, \infty)^{n}\right)$.)

In this $\mathbf{C}^{n}$ case one has the additional useful fact that a holomorphic function $f$ on $\mathbf{C}^{n}$ has small characteristic $T(r, f)=O(\log (r))$ if and only if $f$ is a polynomial. Hence, if $f$ is a polynomial and $g$ is any nonconstant holomorphic function on $\mathbf{C}^{n}$, then $T(r, f)=o\left(T\left(r, e^{g}\right)\right.$ ) (which is necessary as part of the hypotheses of the $\mathrm{H}-\mathrm{O}$ Lemma as we apply it). See [KUJ, Proposition 4.4ff]. For holomorphic functions this can be proved by estimating the Poisson integral for $\log |f|$ much as in the proof of Lemma 2.3 to show that $f$ is of polynomial growth as a function of $x_{j}$ (when $x_{i}, i \neq j$, are held fixed), for each $j=1,2, \ldots, n$. By the Liouville theorem in one variable, then, $f$ is a polynomial separately in each $x_{j}$. That $f$ is globally a polynomial now follows from [PAL]. (There must be many other proofs of our assertion in the literature.) 
THEOREM 5.2 (TARSKI's CONJECTURE FOR $\Sigma$ ). If $t, s$ are any two terms in $\Sigma$ and $t \equiv s$, then the identity $t=s$ is provable from the axioms

$$
\begin{array}{ccrl}
x+(y+z)=(x+y)+z, & x(y z) & =(x y) z, \\
x+y=y+x, & x y & =y x, \\
x+0=x, & 1 \cdot x & =x, \\
x(y+z)=x y+x z, & 0 \cdot x & =0, \\
\exp (x+y)=\exp (x) \cdot \exp (y), &
\end{array}
$$

together with all axioms giving the facts of addition, multiplication and exponentiation for constants from $\mathbf{C}$.

PROOF. Because we have included here a constant for -1 , the operation of subtraction is available and we need only consider the case where $s$ is 0 . That is, if $t \in \Sigma$ then we must show that $t=0$ is formally derivable whenever $t \equiv 0$.

Moreover, it is easy to show that for any term $t \in \Sigma$ there are terms $s_{1}, \ldots, s_{k} \in \Sigma$ and polynomials $p_{1}, \ldots, p_{k}$ in $n$ variables, with coefficients in $\mathbf{C}$ (also realized as terms in $\Sigma$ ) so that the identity

$$
t=p_{1} \cdot \exp \left(s_{1}\right)+\cdots+p_{k} \cdot \exp \left(s_{k}\right)
$$

is provable from the permitted axioms. We will prove the theorem by induction on the total number of symbols in the sequence $s_{1}, \ldots, s_{k}$, showing that if $p_{1}, \ldots, p_{k}$ are polynomials, $s_{1}, \ldots, s_{k} \in \Sigma$ and $p_{1} \exp \left(s_{1}\right)+\cdots+p_{k} \exp \left(s_{k}\right) \equiv 0$, then $p_{1} \cdot \exp \left(s_{1}\right)$ $+\cdots+p_{k} \cdot \exp \left(s_{k}\right)=0$ is formally derivable. (Note we allow $s_{j}$ to be 0 .)

First suppose $k=1$ : if $p_{1} \cdot \exp \left(s_{1}\right) \equiv 0$ then $p_{1} \equiv 0$. It is well known that $p_{1}=0$ is provable from the admitted axioms, since $p_{1}$ is a polynomial. Hence also $p_{1} \exp \left(s_{1}\right)=0$ is provable.

From now on assume $k>1$. Assume $p_{1}, \ldots, p_{k}$ are polynomials, $s_{1}, \ldots, s_{k} \in \Sigma$ and $p_{1} \cdot \exp \left(s_{1}\right)+\cdots+p_{k} \cdot \exp \left(s_{k}\right) \equiv 0$. For $1 \leqslant j \leqslant k$ let $\pi_{j}$ be the function on $\mathbf{C}^{n}$ defined by $\exp \left(s_{j}\right)$. (Choose $n$ so that all variables in each $p_{j}$ and $s_{j}$ are included among $x_{1}, \ldots, x_{n}$.) Note that we may assume each $\pi_{j}$ is nowhere equal to 0 on $\mathbf{C}^{n}$.

After dividing by $\pi_{k}$ we have

$$
p_{1}\left(\pi_{1} / \pi_{k}\right)+\cdots+p_{k-1}\left(\pi_{k-1} / \pi_{k}\right) \equiv-p_{k} .
$$

Suppose first that we can apply the $\mathrm{H}-\mathrm{O}$ Lemma. In this setting, this means $T\left(r, \pi_{i} / \pi_{k}\right) \neq O(\log (r))$ for each $1 \leqslant i \leqslant k-1$. If so, then there exist constants $c_{1}, \ldots, c_{k-1}($ not all 0$)$ so that

$$
c_{1} p_{1}\left(\pi_{1} / \pi_{k}\right)+\cdots+c_{k-1} p_{k-1}\left(\pi_{k-1} / \pi_{k}\right) \equiv 0
$$

which gives us an identity with $k-1$ exponentials after multiplying through by $\pi_{k}$. By the induction hypothesis, the formal identity

$$
c_{1} p_{1} \exp \left(s_{1}\right)+\cdots+c_{k-1} p_{k-1} \exp \left(s_{k-1}\right)=0
$$

is derivable in the allowed system. Now we can use this identity to solve for one of the expressions $p_{j} \cdot \exp \left(s_{j}\right)(1 \leqslant j \leqslant k-1)$ and eliminate it from the original 
expression $p_{1} \exp \left(s_{1}\right)+\cdots+p_{k} \exp \left(s_{k}\right)$. The resulting identity (setting this expression $=0$ ) has at most $k-1$ exponentials, so it is derivable. From this one deduces the desired identity

$$
p_{1} \exp \left(s_{1}\right)+\cdots+p_{k} \exp \left(s_{k}\right)=0 .
$$

On the other hand, it may happen that for some $i(1 \leqslant i \leqslant k-1), T\left(r, \pi_{i} / \pi_{k}\right)=$ $O(\log r)$. Since $\pi_{i}, \pi_{k}$ are nowhere 0 , it follows that $\pi_{i} \equiv c \pi_{k}$ for some constant $c$. (By (C2.3) the same kind of "big- $O$ " estimate holds for $\pi_{k} / \pi_{i}$, and hence both $\pi_{i} / \pi_{k}$ and $\pi_{k} / \pi_{i}$ are polynomials.) That is, $\exp \left(s_{i}\right) \equiv c \cdot \exp \left(s_{k}\right)$ so that for some constant $d \in \mathbf{C}, c=e^{d}$ and $s_{i}-s_{k} \equiv d$. Using the induction hypothesis, we therefore get a formal derivation of $s_{i}-s_{k}-d=0$ and, hence, also of $\exp \left(s_{i}\right)=c \cdot \exp \left(s_{k}\right)$. This allows us to reduce the original identity to one involving only $\exp \left(s_{j}\right)$ for $1 \leqslant j \leqslant$ $k-1$, which will be derivable by the induction hypothesis. Again this yields a derivation of the identity $p_{1} \exp \left(s_{1}\right)+\cdots+p_{k} \exp \left(s_{k}\right)=0$ and completes the proof.

Theorem 5.2 has an interesting corollary for trigonometric functions, which we present next. Consider terms in a language with constants for all the complex numbers, variables $x_{1}, x_{2}, \ldots$ and function symbols for addition and multiplication and for $\sin$ and $\cos$. Let $\Sigma^{*}$ be the set of all these terms.

Corollary 5.3. If $t, s$ are any two terms in $\Sigma^{*}$ and $t \equiv s$, then the identity $t=s$ is provable from the axioms

$$
\begin{array}{cc}
x+(y+z)=(x+y)+z, & x(y z)=(x y) z, \\
x+y=y+x, & x y=y x, \\
x+0=x, & 1 \cdot x=x, \\
x(y+z)=x y+x z, & 0 \cdot x=0, \\
\sin (x+y)=\sin (x) \cos (y)+\cos (x) \sin (y),
\end{array}
$$

together with all axioms giving the facts of addition, multiplication, $\sin$ and cos for constants from $\mathbf{C}$.

Proof. We use the fact that in the context of the complex plane, $e^{x}$ is interdefinable with sin and cos. Note that since the allowed axioms include the identities $\sin (\pi / 2)=1$ and $\cos (\pi / 2)=0$, we can prove $\cos (x)=\sin (x+\pi / 2)$. This in turn allows us to derive the other addition identity,

$$
\cos (x+y)=\cos (x) \cos (y)-\sin (x) \sin (y) .
$$

In $\Sigma^{*}$ let $\operatorname{EXP}(x)$ be an abbreviation for the term $\cos (-i \cdot x)+i \cdot \sin (-i \cdot x)$. It is easy to verify that from the allowed identities in $\Sigma^{*}$ one can prove the exponential identity

$$
\operatorname{EXP}(x+y)=\operatorname{Exp}(x) \cdot \operatorname{Exp}(y)
$$

as well as all the numerical facts involving EXP.

Given any term $t$ in $\Sigma^{*}$, we define a term $t^{\#}$ in $\Sigma$ by replacing (inductively) each term of the form $\sin (s)$ by

$$
-.5 i \cdot(\exp (i \cdot s)-\exp (-i \cdot s))
$$

and $\cos (s)$ by

$$
.5 \cdot(\exp (i \cdot s)+\exp (-i \cdot s))
$$


If $t$ is any term in $\Sigma$ we define $t^{*}$ in $\Sigma^{*}$ by replacing (inductively) each term of the form $\exp (s)$ by $\operatorname{EXP}(s)$. Note that if $t \in \Sigma^{*}$ then the identity $t=\left(t^{\sharp}\right)^{*}$ is provable from the axioms allowed in Corollary 5.3.

Now suppose $t, s \in \Sigma^{*}$ and $t \equiv s$. Then $t^{\#} \equiv s^{\#}$, so the identity $t^{\#}=s^{\#}$ is provable from the axioms allowed in Theorem 5.2. Hence $\left(t^{\#}\right)^{*}=\left(s^{\#}\right)^{*}$ is provable in the system of Corollary 5.3. It follows that $t=s$ is also provable in that system, completing the proof.

REMARK. Suppose $t, s \in \Sigma^{*}$ and $t$, $s$ only contain real constants. We do not know if there is a proof of the identity $t=s$ in the system of Corollary 5.3 in which only real constants appear.

Next we settle positively a conjecture which was communicated to one of us by $\mathrm{S}$. Schanuel.

THEOREM 5.4. Let $t \in \Sigma$ and suppose the function represented by $t$ is nowhere equal to 0 . Then $\log (t)$ is in $\Sigma$, in the sense that $t \equiv e^{s}$ for some $s \in \Sigma$.

Proof. Let $\pi$ be the function (on $\mathbf{C}^{n}$ say) defined by $t$. There is some holomorphic function $G$ on $\mathrm{C}^{n}$ so that $\pi \equiv e^{G}$. We may suppose $t$ is a term of the form $p_{1} \exp \left(s_{1}\right)+\cdots+p_{k} \exp \left(s_{k}\right)$, and we argue by induction on the number of symbols in $s_{1}, \ldots, s_{k}$ as in the proof of Theorem 5.2. Clearly we are done if $k=1$. Assume $k>1$ and for $1 \leqslant j \leqslant k$ let $\pi_{j}$ be the function on $\mathbf{C}^{n}$ defined by $\exp \left(s_{j}\right)$. Then we have $p_{1} \pi_{1}+\cdots+p_{k} \pi_{k} \equiv e^{G}$ so that $p_{1}\left(\pi_{1} e^{-G}\right)+\cdots+p_{k}\left(\pi_{k} e^{-G}\right) \equiv 1$. We may assume the functions $p_{1} \pi_{1} e^{-G}, \ldots, p_{k} \pi_{k} e^{-G}$ are linearly independent (otherwise we could replace $t$ by a simpler term to which the induction hypothesis would apply). Hence the $\mathrm{H}-\mathrm{O}$ Lemma cannot apply. It follows as argued in the proof of Theorem 5.2 that there must exist $1 \leqslant i<j \leqslant k$ so that $\pi_{i} / \pi_{j}$ is identically constant. Again this permits us to reduce the complexity of $t$ and to apply the induction hypothesis. This completes the proof.

REMARK. In this section we have only considered the language in which all constants for complex numbers are included. This does not immediately tell us which constants may be needed in the formal proof of a given (true) identity $t \equiv s$. This information is more available in the form of results such as those in $\S 3$, where a specific set of constants $S$ is considered. Indeed, it is not too difficult to carry out such a more detailed analysis here, although we have chosen not to do so. Also it would be possible to prove results such as Theorems 5.2 and 5.3 when, in addition, the exponential function is taken to have some other base than the number $e$.

\section{REFERENCES}

[BIS] A. Biancofiore and W. Stoll, Another proof of the lemma of the logarithmic derivative in several complex variables, Recent Developments in Several Complex Variables, Princeton Univ. Press, Princeton, N.J., 1981, pp. 29-45.

[BRO] W. D. Brownawell, A measure of linear independence for some exponential functions. II, Canad. J. Math. 31 (1979), 341-346.

[BOS] M. Boshernitzan, An extension of Hardy's class L of “orders of infinity," J. Analyse Math. 39 (1981), 235-255.

[CAR] H. Cartan, Sur les systèmes de fonctions holomorphes d'variétés linéaires lacunaires et leurs applications, Ann. Sci. École Norm. Sup. (3) 64 (1928), 255-346. 
[EHR] A. Ehrenfeucht, Polynomial functions with exponentiation are well-ordered, Algebra Universalis 3 (1973), 261-262.

[GAH] P. M. Gauthier and W. Hengartner, The value distribution of most functions of one or several complex variables, Ann. of Math. (2) 96 (1972), 31-52.

[HAR] G. H. Hardy, Orders of infinity, Cambridge Tracts in Math. and Math. Phys., No. 12, Cambridge Univ. Press, 1910; Reprint, Hafner, New York.

[HAY] W. K. Hayman, Meromorphic functions, Oxford Univ. Press, Oxford, 1964.

[HIO] G. Hiromi and M. Ozawa, On the existence of analytic mappings between two ultrahyperelliptic surfaces, Kōdai Math. Sem. Rep. 17 (1965), 281-306.

[KUJ] R. O. Kujala, Functions of finite $\lambda$-type in several complex variables, Trans. Amer. Math. Soc. 161 (1971), 327-358.

[LEV] H. Levitz, An ordered set of arithmetic functions representing the least \&-number, Z. Math. Logik Grundlag. Math. 21 (1975), 115-120.

[MAC] A. Macintyre, The laws of exponentiation, Model Theory and Arithmetic, Lecture Notes in Math., vol. 890, Springer-Verlag, 1981, pp. 185-197.

[MAR] C. F. Martin, Equational theories of natural numbers and transfinite ordinals, Ph.D. Thesis, Berkeley, 1973.

[NEV I] R. Nevanlinna, Über die Eigenschaften meromorpher Functionen in einem Winkelraum, Acta Soc. Sci. Fenn. 50 (1925).

[NEV II] _ Le théorème de Picard-Borel et la théorie des fonctions méromorphes, Gauthier-Villars, Paris, 1929.

[PAL] R. S. Palais, Some analogues of Hartogs' theorem in an algebraic setting, Amer. J. Math. 100 (1978), 387-405.

[RIC I] D. Richardson, Some undecidable problems involving elementary functions of a real variable, J. Symbolic Logic 33 (1968), 514-520.

[RIC II] _ Solution of the identity problem for integral exponential functions, Z. Math. Logik Grundlagen. Math. 15 (1969), 333-340.

[RON] L. I. Ronkin, Introduction to the theory of entire functions of several variables, Transl. Math. Monographs, vol. 44, Amer. Math. Soc., Providence, R.I., 1974.

[RUD] W. Rudin, Function theory in polydisks, Benjamin, New York, 1969.

[STO] W. Stoll, Value distribution and the lemma of the logarithmic derivative in polydisks, Internat. J. Math. Math. Sci. (to appear).

[TAB] B. A. Taylor, The fields of quotients of some rings of entire functions, Entire Functions and Related Parts of Analysis, Proc. Sympos. Pure Math., vol. 11, Amer. Math. Soc., Providence, R.I., 1968, pp. 468-474.

[TAW] W. Taylor, Equational logic, supplement to Houston J. Math. 1979.

[TSU] M. Tsuji, On Borel's directions of meromorphic functions of finite order. I, Tôhoku Math. J. 2 (1950), 97-112.

[VVD] L. van den Dries, Exponential rings, exponential polynomials and exponential functions (preprint).

[VIT] A. Vitter, The lemma of the logarithmic derivative in several complex variables, Duke Math. J. 44 (1977), 89-104.

[WIL I] A. Wilkie, private communication.

[WIL II] _ On exponentiation—a solution to Tarski's High School Algebra Problem (preprint).

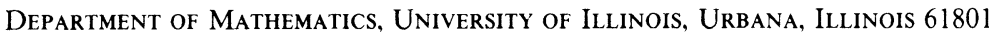

